\title{
Multiresolution Time-Domain Scheme for Terminal Response of Two-Conductor Transmission Lines
}

\author{
Zongliang Tong, Lei Sun, Ying Li, and Jianshu Luo \\ College of Science, National University of Defense Technology, Changsha, Hunan 410073, China \\ Correspondence should be addressed to Zongliang Tong; tongzl@nudt.edu.cn
}

Received 4 January 2016; Revised 20 March 2016; Accepted 18 April 2016

Academic Editor: M. I. Herreros

Copyright (C) 2016 Zongliang Tong et al. This is an open access article distributed under the Creative Commons Attribution License, which permits unrestricted use, distribution, and reproduction in any medium, provided the original work is properly cited.

This paper derives a multiresolution time-domain (MRTD) scheme for the two-conductor lossless transmission line equations based on Daubechies' scaling functions. And a method is proposed to generate the scheme at the terminal and near the terminal of the lines. The stability and numerical dispersion of this scheme are studied, and the proposed scheme shows a better dispersion property than the conventional FDTD method. Then the MRTD scheme is extended to the two-conductor lossy transmission line equations. The MRTD scheme is implemented with different basis functions for both lossless and lossy transmission lines. Numerical results show that the MRTD schemes which use the scaling functions with high vanishing moment obtain more accurate results.

\section{Introduction}

The multiresolution time-domain (MRTD) scheme proposed by [1] provides an efficient algorithm for electromagnetic field computation and shows excellent capability to approximate exact solution with low sampling rates. However, the BattleLemarie wavelet function used in [1] is not compact supported, which means the iterative equations contain infinite terms. We must cut off the iterative equations in the actual computation and this may introduce truncation errors. So different wavelet bases, which are compact supported with some numbers of vanishing moments, have been used to improve this method [2-5]. This makes a great development for MRTD schemes. As a kind of numerical method, the MRTD schemes show great advantages in numerical dispersion properties [6-9]; meanwhile, these schemes need a more rigorous stable condition than the conventional FDTD method [10]. For containing more terms in the iterative equations, the terminal conditions or absorbing boundary conditions are more complicated to process in MRTD schemes; this disadvantage has limited the application of the MRTD scheme. To overcome this limitation, some works on the perfect match layer have been made [11-13]; however, other terminal conditions also need to be analyzed specifically. For the transmission lines equations, the resistive terminal conditions could be equivalent as a general Thevenin circuit; this paper will solve this kind of terminal condition in the MRTD scheme.

Since the appearance of the telegraph equations, studies on transmission lines have had a considerable development. Several equivalent forms of transmission line theory have been proposed to describe the influence of the incident electromagnetic field to the transmission lines [14-16]. In [17], the classical theory of the transmission line has been summarized and the theory on the high frequency radiation effects to the transmission lines is introduced. In the monograph [18], the multiconductor transmission lines (MTL) theory has been comprehensively studied in detail. For the twoconductor lossless transmission lines, there are several methods, which contain the series solution, the SPICE solution, the time-domain to frequency-domain (TDFD) transformation method, and the FDTD method [18]. However, the MRTD scheme has not been used to calculate the terminal response of transmission lines. In this paper we will derive a MRTD scheme for this problem.

In this paper, we focus on the calculation of the terminal response of two-conductor transmission lines equations by using MRTD scheme. In Section 2, the MRTD scheme is derived based on Daubechies' scaling functions for the two-conductor lossless transmission line equations, and, for 
the resistive terminations, the iterative equations for the terminal voltages are derived, a method is proposed to update the iterative equations which contain some terms whose indices exceed the index range in the MRTD scheme, and then the stability and the numerical dispersion are studied. In Section 3, the MRTD scheme is extended to the two-conductor lossy transmission line. In Section 4, the numerical results are presented on the terminal response of both lossless and lossy transmission lines using the MRTD scheme and compared to the FDTD method at different space discretization numbers and different Courant numbers.

\section{MRTD Scheme for Two-Conductor Lossless Transmission Lines}

2.1. MRTD Formulation. In this section, the MRTD scheme is applied to the following scalar transmission lines equations for two-conductor lossless lines [18]:

$$
\begin{aligned}
& \frac{\partial V(z, t)}{\partial z}+l \frac{\partial I(z, t)}{\partial t}=0 \\
& \frac{\partial I(z, t)}{\partial z}+c \frac{\partial V(z, t)}{\partial t}=0
\end{aligned}
$$

where $l$ and $c$ are the per-unit-length inductance and capacitance, respectively.

Based on the method outlined in [1], the voltage and current can be expanded as follows:

$$
\begin{aligned}
V(z, t) & =\sum_{k, n=-\infty}^{+\infty} V_{k}^{n} \phi_{k}(z) h_{n}(t) \\
I(z, t) & =\sum_{k, n=-\infty}^{+\infty} I_{k+1 / 2}^{n+1 / 2} \phi_{k+1 / 2}(z) h_{n+1 / 2}(t),
\end{aligned}
$$

where $V_{k}^{n}$ and $I_{k+1 / 2}^{n+1 / 2}$ are the coefficients for the voltages and currents in terms of scaling functions, respectively. The indices $n$ and $k$ are the discrete spatial and temporal indices related to space and time coordinates via $z=k \Delta z$ and $t=n \Delta t$, where $\Delta z$ and $\Delta t$ represent the spatial and temporal discretization intervals in $z$ and $t$ direction. The function $h_{n}(t)$ is defined as

$$
h_{n}(t)=h\left(\frac{t}{\Delta t}-n\right)
$$

with the rectangular pulse function

$$
h(t)= \begin{cases}1 & \text { for }|t|<\frac{1}{2} \\ \frac{1}{2} & \text { for }|t|=\frac{1}{2} \\ 0 & \text { for }|t|>\frac{1}{2}\end{cases}
$$

The function $\phi_{k}(z)$ is defined as

$$
\phi_{k}(z)=\phi\left(\frac{z}{\Delta z}-k\right),
$$

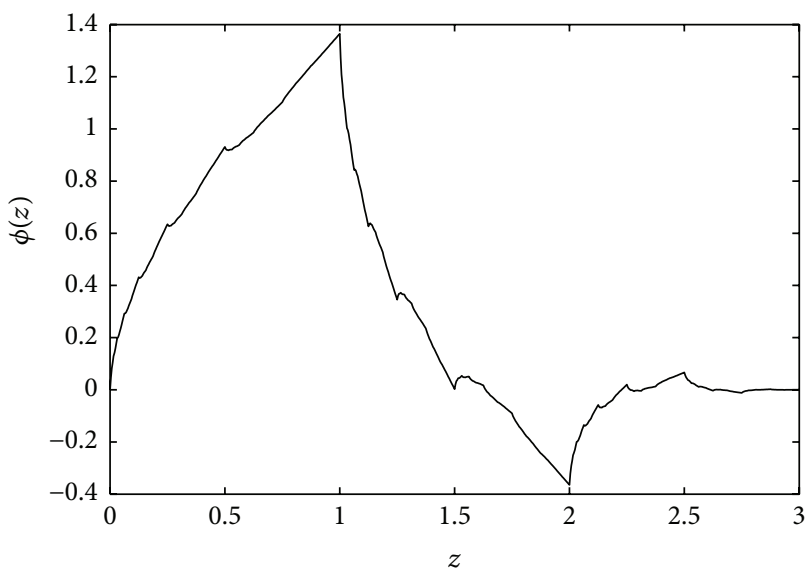

FIgURE 1: Daubechies' scaling function with two vanishing moments.

where $\phi(z)$ represents Daubechies'scaling function. Figure 1 shows Daubechies' scaling function with two vanishing moments.

For deriving the MRTD scheme for (1a) and (1b), we need the following integrals:

$$
\begin{gathered}
\int_{-\infty}^{+\infty} h_{n}(t) h_{n^{\prime}}(t) d t=\delta_{n, n^{\prime}} \Delta t \\
\int_{-\infty}^{+\infty} h_{n}(t) \frac{\partial h_{n^{\prime}+1 / 2}(t)}{\partial t} d t=\delta_{n, n^{\prime}}-\delta_{n, n^{\prime}+1}
\end{gathered}
$$

where $\delta_{n, n^{\prime}}$ represents the Kronecker symbol. Consider

$$
\begin{gathered}
\int_{-\infty}^{+\infty} \phi_{k}(z) \phi_{k^{\prime}}(z) d z=\delta_{k, k^{\prime}} \Delta z \\
\int_{-\infty}^{+\infty} \phi_{n}(z) \frac{\partial \phi_{n^{\prime}+1 / 2}(z)}{\partial z} d z=\sum_{i=-L_{S}}^{L_{S}-1} a(i) \delta_{n+i, n^{\prime}}
\end{gathered}
$$

where $L_{S}$ denotes the effective support size of the basis functions. The coefficients $a(i)$ are called connection coefficients and can be calculated by (9). Taking Daubechies' scaling functions as the basis functions, Table 1 shows $a(i)$ for $0 \leq$ $i \leq L_{S}-1$, which are zeros for $i>L_{S}-1$, and for $i<0$ it can be obtained by the symmetry relation $a(-1-i)=-a(i)$,

$$
a(i)=\frac{1}{\pi} \int_{0}^{\infty}|\widehat{\phi}(\lambda)|^{2} \lambda \sin \lambda\left(i+\frac{1}{2}\right) d \lambda,
$$

where $\widehat{\phi}(\lambda)$ represents the Fourier transform of $\phi(z)$.

Daubechies' scaling functions satisfy the shifted interpolation property [19]

$$
\phi\left(i+M_{1}\right)=\delta_{i, 0}
$$

for $i$ integer, where $M_{1}=\int_{-\infty}^{+\infty} z \phi(z) d z$ is the first moment of the scaling functions and the values of $M_{1}$ are listed in Table 1.

Following the theory in [3] and making use of (10), (5) is modified to

$$
\phi_{k}(z)=\phi\left(\frac{z}{\Delta z}-k+M_{1}\right)
$$


TABLE 1: Connection coefficients $a(i)$ and the first-order moments $M_{1}$ of Daubechies' scaling functions.

\begin{tabular}{lccc}
\hline$i$ & $D_{2}$ & $D_{3}$ & $D_{4}$ \\
\hline 0 & 1.2291666667 & 1.2918129281 & 1.3110340773 \\
1 & -0.0937500000 & -0.1371343465 & -0.1560100110 \\
2 & 0.0104166667 & 0.0287617728 & 0.0419957460 \\
3 & & -0.0034701413 & -0.0086543236 \\
4 & & 0.0000080265 & 0.0008308695 \\
5 & & & 0.0000108999 \\
6 & & & 0.0000000041 \\
\hline$M_{1}$ & 0.6339743121 & 0.8174005815 & 1.0053923835 \\
\hline$q_{\max }$ & 0.7500 & 0.6844 & 0.6585 \\
\hline
\end{tabular}

In spite of the support of the scaling functions [20], singlepoint sampling of the total voltages and currents can be taken at integer points with negligible error. Taking voltage at spatial point $k \Delta z$ and at time $n \Delta t$, we obtain

$$
V(k \Delta z, n \Delta t)
$$

$$
\begin{aligned}
& =\iint_{-\infty}^{+\infty} V(z, t) \delta\left(\frac{z}{\Delta z}-k\right) \delta\left(\frac{t}{\Delta t}-n\right) d z d t \\
& =V_{k}^{n}
\end{aligned}
$$

where $\delta$ is the Dirac delta function. Equation (12) means the voltage value at each integer point is equal to the coefficient. The current values have the same character at each half integer point. Therefore, we will use $V_{k}^{n}$ and $I_{k+1 / 2}^{n+1 / 2}$ directly to represent the voltage at the point $(k \Delta z, n \Delta t)$ and the current at the point $((k+1 / 2) \Delta z,(n+1 / 2) \Delta t)$ in this paper.

The modified $\phi_{k}(z)$ in (11) also satisfy integrals (7) and (8). Applying the Galerkin technique to (1a) and (1b), we can obtain the following iterative equations for the voltages and currents:

$$
\begin{aligned}
& V_{k}^{n+1}=V_{k}^{n}-\frac{\Delta t}{\Delta z} c^{-1} \sum_{i=0}^{L_{S}-1} a(i)\left(I_{k+i+1 / 2}^{n+1 / 2}-I_{k-i-1 / 2}^{n+1 / 2}\right) \\
& I_{k+1 / 2}^{n+1 / 2}=I_{k+1 / 2}^{n-1 / 2}-\frac{\Delta t}{\Delta z} l^{-1} \sum_{i=0}^{L_{S}-1} a(i)\left(V_{k+i+1}^{n}-V_{k-i}^{n}\right) .
\end{aligned}
$$

2.2. Terminal Iterative Equations for Resistive Load in MRTD Scheme. We will consider the terminal conditions for the two-conductor lossless transmission lines equations in this section. Equations (1a) and (1b) are homogeneous linear equations; we need to add the terminal conditions to obtain the unique solution.

Considering the two-conductor lines shown in Figure 2, we assume the length of the total line is $L$ and the resistive loads are $R_{S}$ and $R_{L}$. The line is divided uniformly into NDZ segments with the space interval $\Delta z$ and the total solution time is divided into NDT steps with the uniform time interval $\Delta t$. Similar to the conventional FDTD, we will calculate the interlace voltages, $V_{0}^{n}, V_{1}^{n}, \ldots, V_{\mathrm{NDZ}}^{n}$, and currents, $I_{1 / 2}^{n+1 / 2}, I_{3 / 2}^{n+1 / 2}, \ldots, I_{\mathrm{NDZ}-1 / 2}^{n+1 / 2}$, in both space domain

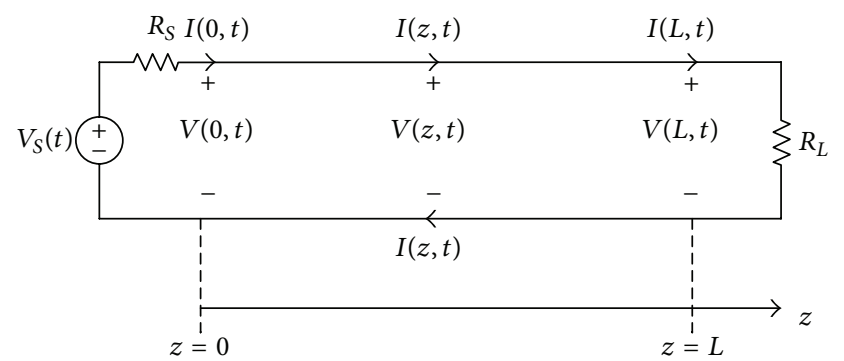

Figure 2: A two-conductor line in time-domain.

and time-domain as shown in (13a) and (13b), for $n=$ $1,2, \ldots$, NDT.

For the resistive terminations, we note the voltage at the source $(z=0)$ as $V_{S}(t)$ and the current at the source as $I_{S}(t)$, the external voltage at the load $(z=L)$ as $V_{L}(t)$, and the current at the load as $I_{L}(t)$. The discrete voltages and currents at the source are denoted as $V_{S}^{n} \equiv V_{S}(n \Delta t)$ and $I_{S}^{n} \equiv I_{S}(n \Delta t)$, and the discrete voltages and currents at the load are denoted as $V_{L}^{n} \equiv V_{L}(n \Delta t)$, and $I_{L}^{n} \equiv I_{L}(n \Delta t)$, then the terminal characterizations could be written in terms of a generalized Thevenin equivalent as

$$
\begin{aligned}
V_{0}^{n} & =V_{S}^{n}-R_{S} I_{S}^{n} \\
V_{\mathrm{NDZ}}^{n} & =V_{L}^{n}+R_{L} I_{L}^{n} .
\end{aligned}
$$

Equations (14a) and (14b) denote the discretization terminal conditions for the case of resistive terminations, so we need to introduce these conditions to the iterative equations (13a) and (13b) to obtain the numerical solution.

Notice that, in the iterative equations (13a) and (13b), not only the iterative equations of the terminal voltages $V_{0}^{n+1}$ and $V_{\mathrm{NDZ}}^{n+1}$ should be derived and the iterative equations of voltages and currents "near" the terminals also need to be updated. The voltages and currents "near" the terminals we mean are the voltages $V_{i}^{n+1}$ and $V_{\mathrm{NDZ}-i}^{n}$ for $i=1,2, \ldots, L_{S}-1$, and the currents $I_{i+1 / 2}^{n+1 / 2}$ and $I_{\mathrm{NDZ}-i+1 / 2}^{n+1 / 2}$ for $i=0,1, \ldots, L_{S}-2$. All of these voltages and currents contain some terms that exceed the index range in iterative equations (13a) and (13b). Figure 3 shows the discretization of the terminal voltages and the voltages and currents near the terminal.

We will derive the MRTD scheme at the terminal firstly. For updating the iterative equations for the terminal voltages, we need to decompose iterative equations ((13a), (13b)). Since the coefficients $a(i)$ satisfy the following relation [4]

$$
\sum_{i=0}^{L_{S}-1}(2 i+1) a(i)=1
$$

substituting (15) into (13a), we can obtain

$$
\begin{gathered}
\sum_{i=0}^{L_{S}-1} a(i)(2 i+1) V_{k}^{n+1}=\sum_{i=0}^{L_{S}-1} a(i)(2 i+1) V_{k}^{n} \\
-\sum_{i=0}^{L_{S}-1} \frac{\Delta t}{(2 i+1) \Delta z} c^{-1}[a(i)(2 i+1) \\
\left.\cdot\left(I_{k+i+1 / 2}^{n+1 / 2}-I_{k-i-1 / 2}^{n+1 / 2}\right)\right] .
\end{gathered}
$$




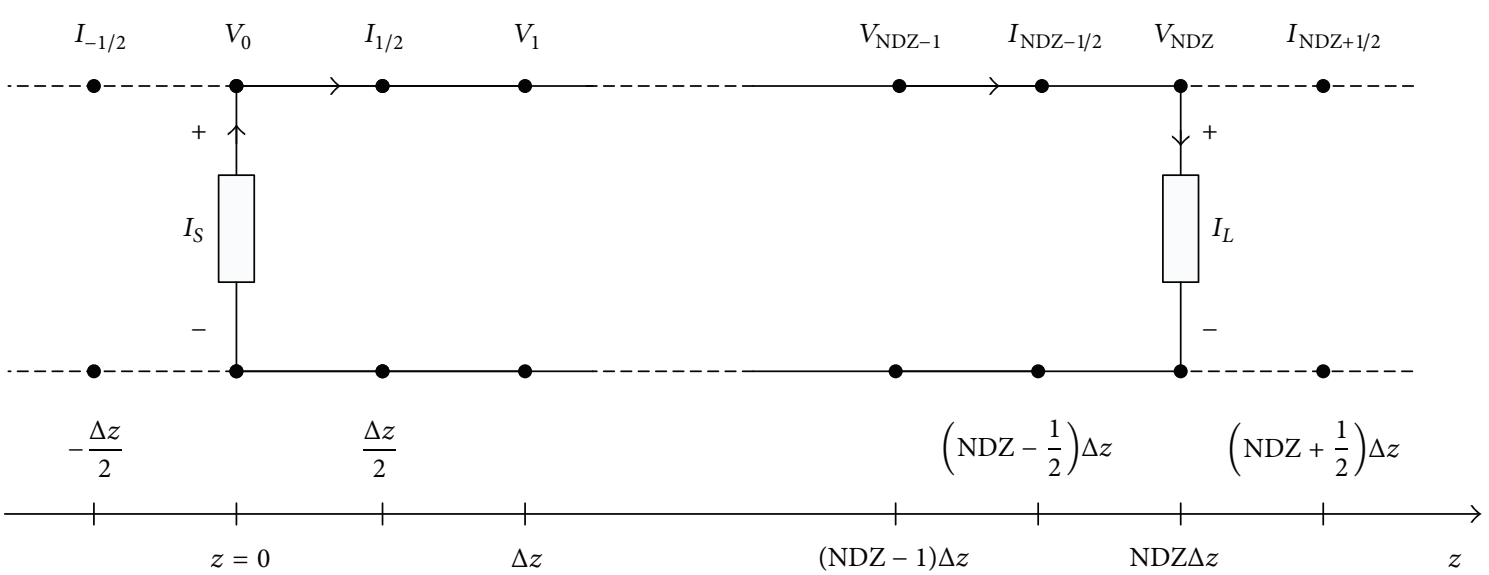

FIGURE 3: Discretizing the terminal voltages and currents.

Considering the corresponding terms with $i$, we can decompose (13a) as [21]

$$
\begin{aligned}
a(i) & (2 i+1) V_{k}^{n+1} \\
= & a(i)(2 i+1) V_{k}^{n} \\
& -a(i)(2 i+1) \frac{\Delta t}{(2 i+1) \Delta z} c^{-1}\left(I_{k+i+1 / 2}^{n+1 / 2}-I_{k-i-1 / 2}^{n+1 / 2}\right)
\end{aligned}
$$

for $i=0,1, \ldots, L_{S}-1$. Equation (13b) could make the analogous decomposition. We could view the MRTD scheme for two-conductor transmission lines as the weighted mean of the conventional FDTD method with spatial discretization step $(2 i+1) \Delta z$ for $i=0,1, \ldots, L_{S}-1$, and the weighting coefficient for each term is $(2 i+1) a(i)$. Besides, for the MRTD scheme whose coefficients $a(i)$ satisfy relationship (15), the analogous decomposition could be made. This relationship between the MRTD scheme and the conventional FDTD method is useful for us to update the iterative equations.

Taking $V_{0}^{n+1}$ as an example to derive the iterative equations at the terminal,

$$
V_{0}^{n+1}=V_{0}^{n}-\frac{\Delta t}{\Delta z} c^{-1} \sum_{i=0}^{L_{S}-1} a(i)\left(I_{i+1 / 2}^{n+1 / 2}-I_{-i-1 / 2}^{n+1 / 2}\right) .
$$
as

Following steps of (16) and (17), we can decompose (18)

$$
\begin{gathered}
a(0) V_{0}^{n+1}=a(0) V_{0}^{n}-a(0) \frac{\Delta t}{\Delta z} c^{-1}\left(I_{1 / 2}^{n+1 / 2}-I_{-1 / 2}^{n+1 / 2}\right) \\
3 a(1) V_{0}^{n+1}=3 a(1) V_{0}^{n}-3 a(1) \frac{\Delta t}{3 \Delta z} c^{-1}\left(I_{3 / 2}^{n+1 / 2}-I_{-3 / 2}^{n+1 / 2}\right) \\
\vdots \\
\left(2 L_{S}-1\right) a\left(L_{S}-1\right) V_{0}^{n+1}=\left(2 L_{S}-1\right) a\left(L_{S}-1\right) V_{0}^{n} \\
-\left(2 L_{S}-1\right) a\left(L_{S}-1\right) \frac{\Delta t}{\left(2 L_{S}-1\right) \Delta z} \\
\cdot c^{-1}\left(I_{L_{S}-1 / 2}^{n+1 / 2}-I_{-L_{S}+1 / 2}^{n+1 / 2}\right) .
\end{gathered}
$$

Here, we could view each equation in (19a), (19b), and (19c) as a central difference scheme, (19a) is the central difference scheme related to points $z=-\Delta z / 2$ and $z=$ $\Delta z / 2,(19 \mathrm{~b})$ is the central difference scheme related to points $z=-3 \Delta z / 2$ and $z=3 \Delta z / 2$, and so on, but the terms $I_{-1 / 2}^{n+1 / 2}, I_{-3 / 2}^{n+1 / 2}, \ldots, I_{-L_{S}+1 / 2}^{n+1 / 2}$, whose subscripts exceed the index range, make (19a), (19b), and (19c) out of work. So we need to make some update for the iterative equations. Using the forward difference scheme to replace the central difference scheme, we change the difference points in (19a) to become $z=0$ and $z=\Delta z / 2$ and change the difference points in (19b) to become $z=0$ and $z=3 \Delta z / 2$; the others follow the same step. Keeping the weighting coefficient unchanged in each equation, we can obtain

$$
\begin{gathered}
a(0) V_{0}^{n+1}=a(0) V_{0}^{n}-a(0) \frac{\Delta t}{\Delta z / 2} c^{-1}\left(I_{1 / 2}^{n+1 / 2}-I_{S}^{n+1 / 2}\right) \\
3 a(1) V_{0}^{n+1}=3 a(1) V_{0}^{n}-3 a(1) \frac{\Delta t}{(3 \Delta z) / 2} c^{-1}\left(I_{3 / 2}^{n+1 / 2}-I_{S}^{n+1 / 2}\right) \\
\vdots \\
\left(2 L_{S}-1\right) a\left(L_{S}-1\right) V_{0}^{n+1}=\left(2 L_{S}-1\right) a\left(L_{S}-1\right) V_{0}^{n} \\
-\left(2 L_{S}-1\right) a\left(L_{S}-1\right) \frac{\Delta t}{\left(2 L_{S}-1\right) \Delta z / 2} \\
\cdot c^{-1}\left(I_{L_{S}-1 / 2}^{n+1 / 2}-I_{S}^{n+1 / 2}\right),
\end{gathered}
$$

where the terminal current $I_{S}^{n+1 / 2}=\left(I_{S}^{n}+I_{S}^{n+1}\right) / 2$ and $I_{S}^{n}$ can be derived from (14a)

$$
I_{S}^{n}=\frac{\left(V_{S}^{n}-V_{0}^{n}\right)}{R_{S}} .
$$

Summing up all the equations in (20a), (20b), and (20c),

$$
V_{0}^{n+1}=V_{0}^{n}-\frac{\Delta t}{\Delta z} c^{-1} \sum_{i=0}^{L_{S}-1} 2 a(i)\left(I_{i+1 / 2}^{n+1 / 2}-I_{S}^{n+1 / 2}\right) .
$$


Substituting (21) into (22), we can obtain the iterative equation at the source

$$
\begin{aligned}
V_{0}^{n+1} & =\left(\sum_{i=0}^{L_{S}-1} a(i)+\frac{\Delta z}{\Delta t} c R_{S}\right)^{-1} \\
\cdot & {\left[\left(\frac{\Delta z}{\Delta t} c R_{S}-\sum_{i=0}^{L_{S}-1} a(i)\right) V_{0}^{n}-2 R_{S} \sum_{i=0}^{L_{S}-1} a(i) I_{i+1 / 2}^{n+1 / 2}\right.} \\
+ & \left.\sum_{i=0}^{L_{S}-1} a(i)\left(V_{S}^{n+1}+V_{S}^{n}\right)\right] .
\end{aligned}
$$

With the same steps, we can obtain the iterative equation at the load

$$
\begin{aligned}
V_{\mathrm{NDZ}}^{n+1} & =\left(\sum_{i=0}^{L_{S}-1} a(i)+\frac{\Delta z}{\Delta t} c R_{L}\right)^{-1} \\
\cdot & {\left[\left(\frac{\Delta z}{\Delta t} c R_{L}-\sum_{i=0}^{L_{S^{-1}}} a(i)\right) V_{\mathrm{NDZ}}^{n}\right.} \\
+ & \left.2 R_{L} \sum_{i=0}^{L_{S}-1} a(i) I_{\mathrm{NDZ}-i+1 / 2}^{n+1 / 2}+\sum_{i=0}^{L_{S}-1} a(i)\left(V_{L}^{n+1}+V_{L}^{n}\right)\right] .
\end{aligned}
$$

After deriving the iterative equations at the terminal, we will put forward a truncation method to update iterative equations which contain some terms whose indices exceed the index range in the MRTD scheme.

Taking $V_{k}^{n+1}$ as an example, for $k=1,2, \ldots, L_{S}-1$, decomposing (13a),

$$
\begin{gathered}
a(0) V_{k}^{n+1}=a(0) V_{k}^{n}-a(0) \frac{\Delta t}{\Delta z} c^{-1}\left(I_{k+1 / 2}^{n+1 / 2}-I_{k-1 / 2}^{n+1 / 2}\right) \\
3 a(1) V_{k}^{n+1}=3 a(1) V_{k}^{n}-3 a(1) \frac{\Delta t}{3 \Delta z} c^{-1}\left(I_{k+3 / 2}^{n+1 / 2}-I_{k-3 / 2}^{n+1 / 2}\right) \\
\vdots \\
(2 k-1) a(k-1) V_{k-1}^{n+1}=(2 k-1) a(k-1) V_{k-1}^{n} \\
-(2 k-1) a(k-1) \frac{\Delta t}{(2 k-1) \Delta z} c^{-1}\left(I_{2 k-1 / 2}^{n+1 / 2}-I_{1 / 2}^{n+1 / 2}\right) \\
(2 k+1) a(k) V_{k}^{n+1}=(2 k+1) a(k) V_{k}^{n}-(2 k+1) a(k) \\
\cdot \frac{\Delta t}{(2 k+1) \Delta z} c^{-1}\left(I_{2 k+1 / 2}^{n+1 / 2}-I_{-1 / 2}^{n+1 / 2}\right) \\
\left(2 L_{S}-1\right) a\left(L_{S}-1\right) V_{L_{S}-1}^{n+1}=\left(2 L_{S}-1\right) a\left(L_{S}-1\right) V_{L_{S}-1}^{n} \\
-\left(2 L_{S}-1\right) a\left(L_{S}-1\right) \frac{\Delta t}{\left(2 L_{S}-1\right) \Delta z} \\
-1\left(I_{k+L_{S}-3 / 2}^{n+1 / 2}-I_{k-L_{S}+1 / 2}^{n+1 / 2}\right) .
\end{gathered}
$$

Noticing the first $k$ terms in (25a), (25b), (25c), (25d), and $(25 \mathrm{e})$, there is no term exceeding the index range in each equation. Meanwhile, the equations which contain the exceeding indices terms are all appearing in the rest of $L_{S}-k$ terms. As mentioned before, we can view the MRTD scheme as the weighted mean of the conventional FDTD method, but (25a), (25b), (25c), (25d), and (25e) show that the last $L_{S}-k$ equations are unavailable for forming the iterative equations in MRTD scheme. To solve this problem, we make truncation here. We update the iterative equation of $V_{k}^{n+1}$ by using the summation of the first $k$ terms in (25a), (25b), (25c), (25d), and (25e) that means we use the weighted mean of the first $k$ to approximate the summation of all the $L_{S}$ terms.

Summing up the first $k$ terms in (25a), (25b), (25c), (25d), and (25e), we can obtain the modified iterative equations

$$
\begin{aligned}
V_{k}^{n+1}=V_{k}^{n} & -\left(\sum_{i=0}^{k-1}(2 i+1) a(i)\right)^{-1} \frac{\Delta t}{\Delta z} \\
& \cdot c^{-1} \sum_{i=0}^{k-1} a(i)\left(I_{k+i+1 / 2}^{n+1 / 2}-I_{k-i-1 / 2}^{n+1 / 2}\right)
\end{aligned}
$$

for $k=1,2, \ldots, L_{S}-1$.

Using the same method, we can obtain the modified iterative equations near the load

$$
\begin{array}{r}
V_{k}^{n+1}=V_{k}^{n}-\left(\sum_{i=0}^{\mathrm{NDZ}-k-1}(2 i+1) a(i)\right)^{-1} \frac{\Delta t}{\Delta z} \\
\cdot c^{-1} \sum_{i=0}^{\mathrm{NDZ}-k-1} a(i)\left(I_{k+i+1 / 2}^{n+1 / 2}-I_{k-i-1 / 2}^{n+1 / 2}\right)
\end{array}
$$

for $k=\mathrm{NDZ}-L_{S}+1, \mathrm{NDZ}-L_{S}+2, \ldots, \mathrm{NDZ}-1$. (13a)

The voltages at the interior points are determined from

$$
V_{k}^{n+1}=V_{k}^{n}-\frac{\Delta t}{\Delta z} c^{-1} \sum_{i=0}^{L_{S}-1} a(i)\left(I_{k+i+1 / 2}^{n+1 / 2}-I_{k-i-1 / 2}^{n+1 / 2}\right)
$$

for $k=L_{S}, L_{S}+1, \ldots, \mathrm{NDZ}-L_{S}$.

For the iterative equation of the current, there is a little difference from the voltage's. As shown in Figure 3, the interlace currents appear at the half integer points which means all the currents are located at the interior points. So we only need to modify the currents near the terminals. Following the same steps of the derivation of voltages iterative equations near the terminal, we could obtain the current iterative equations near the terminals.

For the current iterative equations near the source

$$
\begin{gathered}
I_{k+1 / 2}^{n+1 / 2}=I_{k+1 / 2}^{n-1 / 2}-\left(\sum_{i=0}^{k}(2 i+1) a(i)\right)^{-1} \frac{\Delta t}{\Delta z} \\
\cdot l^{-1} \sum_{i=0}^{k} a(i)\left(V_{k+i+1}^{n}-V_{k-i}^{n}\right)
\end{gathered}
$$

for $k=0,1, \ldots, L_{S}-2$. 


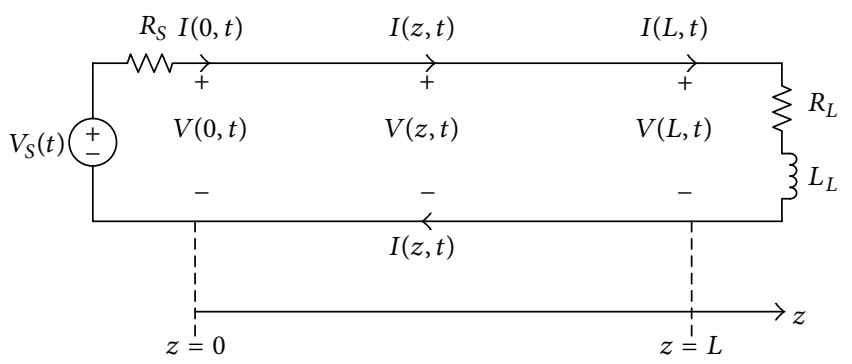

FIGURE 4: A two-conductor line with an inductive resistance.

For the current iterative equations near the load

$$
\begin{gathered}
I_{k+1 / 2}^{n+1 / 2}=I_{k+1 / 2}^{n-1 / 2}-\left(\sum_{i=0}^{\mathrm{NDZ}-k-1}(2 i+1) a(i)\right)^{-1} \frac{\Delta t}{\Delta z} \\
\cdot l^{-1} \sum_{i=0}^{\mathrm{NDZ}-k-1} a(i)\left(V_{k+i+1}^{n}-V_{k-i}^{n}\right)
\end{gathered}
$$

for $k=\mathrm{NDZ}-L_{S}+1, \mathrm{NDZ}-L_{S}+2, \ldots, \mathrm{NDZ}-1$. (13b)

The currents at the interior points are determined from

$$
I_{k+1 / 2}^{n+1 / 2}=I_{k+1 / 2}^{n-1 / 2}-\frac{\Delta t}{\Delta z} l^{-1} \sum_{i=0}^{L_{S^{-}}} a(i)\left(V_{k+i+1}^{n}-V_{k-i}^{n}\right)
$$

for $k=L_{S}-1, L_{S}, \ldots, \mathrm{NDZ}-L_{S}$.

2.3. Terminal Iterative Equations for Inductive Resistance in $M R T D$ Scheme. Since we have discussed the resistive load in Section 2.2, we will consider a more complicated terminal load which consisted of a resistance and inductance shown in Figure 4. The resistance and the inductance at the load are noted as $R_{L}$ and $L_{L}$, respectively.

Keeping the source as a resistive terminal and changing the load including inductance, we note the voltage at the source $(z=0)$ as $V_{S}(t)$ and the current at the source as $I_{S}(t)$, the external voltage at the load $(z=L)$ as $V_{L}(t)$, and the current at the load as $I_{L}(t)$. The terminal conditions could be written as follows:

$$
\begin{aligned}
V_{0}(t) & =V_{S}(t)-R_{S} I_{S}(t) \\
V_{\mathrm{NDZ}}(t) & =V_{L}(t)+R_{L} I_{L}(t)+L_{L} \frac{d I_{L}(t)}{d t} .
\end{aligned}
$$

Expanding $V_{0}(t), I_{0}(t), V_{L}(t)$, and $I_{L}(t)$ as $(2 \mathrm{a})$ and $(2 \mathrm{~b})$ and sampling them at the time discreting point $(n+1 / 2) \Delta t$, the terminal conditions become

$$
\begin{aligned}
\frac{V_{0}^{n+1}+V_{0}^{n}}{2}= & \frac{V_{S}^{n+1}+V_{S}^{n}}{2}-R_{S} I_{S}^{n+1 / 2} \\
\frac{V_{\mathrm{NDZ}}^{n+1}+V_{\mathrm{NDZ}}^{n}}{2}= & \frac{V_{L}^{n+1}+V_{L}^{n}}{2}+R_{L} I_{L}^{n+1 / 2} \\
& +\frac{L_{L}}{2 \Delta t}\left(I_{L}^{n+3 / 2}-I_{L}^{n-1 / 2}\right) .
\end{aligned}
$$

It could be seen from Section 2.2 that the change of the terminal condition only affects the iterative equations at the terminals, so we just need to consider two iterative equations. Since we keep the source as a resistive termination, the iterative equation at the source should be the same as (23). Actually, if we substitute (33a) into (22), we could obtain (23). So the only iterative equation we should derive is located at the load. If we set $L_{L}=0$ in (33b), the terminal condition at the load will have the analogous form with the source, which means the load with a resistance and inductance degenerates to be a resistance. For those $L_{L} \neq 0$, transforming (33b) as

$$
\begin{aligned}
& I_{L}^{n+3 / 2}=I_{L}^{n-1 / 2}+\frac{\Delta t}{L_{L}}\left(\left(V_{\mathrm{NDZ}}^{n+1}+V_{\mathrm{NDZ}}^{n}\right)-\left(V_{L}^{n+1}+V_{L}^{n}\right)\right. \\
& \left.\quad-2 R_{L} I_{L}^{n+1 / 2}\right) .
\end{aligned}
$$

Following the steps we get the iterative equation of $V_{0}^{n+1}$ in Section 2.2; we can obtain the iterative equation at the load

$$
\begin{aligned}
V_{\mathrm{NDZ}}^{n+1}= & V_{\mathrm{NDZ}}^{n} \\
& -\frac{\Delta t}{\Delta z} c^{-1} \sum_{i=0}^{L_{S}-1} 2 a(i)\left(I_{\mathrm{NDZ}-i+1 / 2}^{n+1 / 2}-I_{L}^{n+1 / 2}\right),
\end{aligned}
$$

where

$$
\begin{aligned}
& I_{L}^{n+1 / 2}=I_{L}^{n-3 / 2}+\frac{\Delta t}{L_{L}}\left(\left(V_{\mathrm{NDZ}}^{n}+V_{\mathrm{NDZ}}^{n-1}\right)-\left(V_{L}^{n}+V_{L}^{n-1}\right)\right. \\
& \left.\quad-2 R_{L} I_{L}^{n-1 / 2}\right)
\end{aligned}
$$

for $n=2,3, \ldots$, NDT.

2.4. Stability Analysis. For the purpose of stability analysis, (13a) and (13b) can be rewritten as

$$
\begin{aligned}
\frac{V_{k}^{n+1}-V_{k}^{n}}{\Delta t} & =-\frac{1}{c \Delta z} \sum_{i=0}^{L_{S}-1} a(i)\left(I_{k+i+1 / 2}^{n+1 / 2}-I_{k-i-1 / 2}^{n+1 / 2}\right) \\
\frac{I_{k+1 / 2}^{n+1 / 2}-I_{k+1 / 2}^{n-1 / 2}}{\Delta t} & =-\frac{1}{l \Delta z} \sum_{i=0}^{L_{S}-1} a(i)\left(V_{k+i+1}^{n}-V_{k-i}^{n}\right) .
\end{aligned}
$$

Following the procedures in [22], the finite-difference approximations of the time derivations on the left side of (37a) and (37b) can be written as an eigenvalue problem

$$
\begin{aligned}
\frac{V_{k}^{n+1}-V_{k}^{n}}{\Delta t} & =\lambda V_{k}^{n+1 / 2} \\
\frac{I_{k+1 / 2}^{n+1 / 2}-I_{k+1 / 2}^{n-1 / 2}}{\Delta t} & =\lambda I_{k+1 / 2}^{n} .
\end{aligned}
$$

In order to avoid instability during normal time stepping, the imaginary part of $\lambda$ must satisfy

$$
|\operatorname{Im}(\lambda)| \leq \frac{2}{\Delta t} .
$$




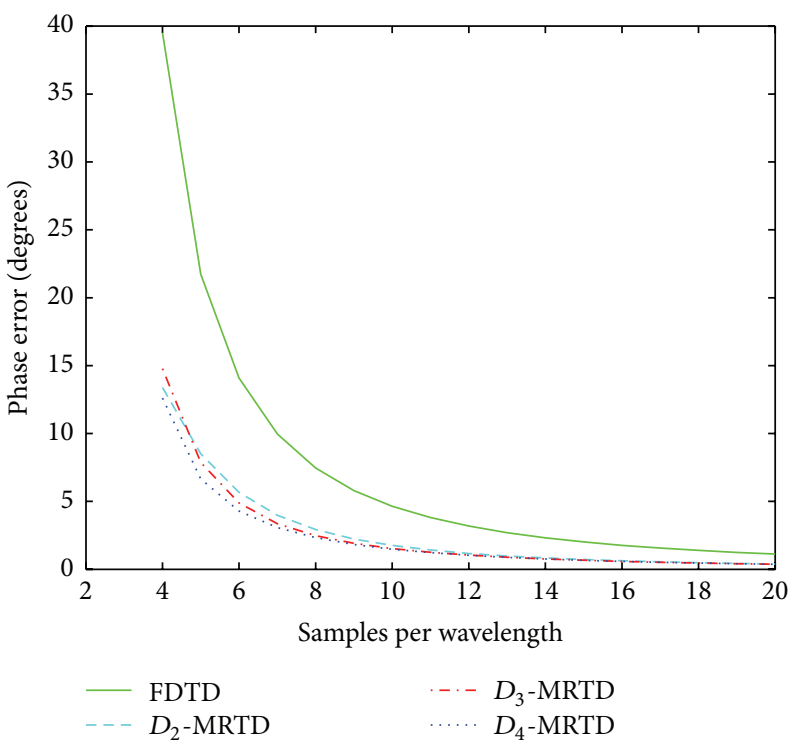

(a)

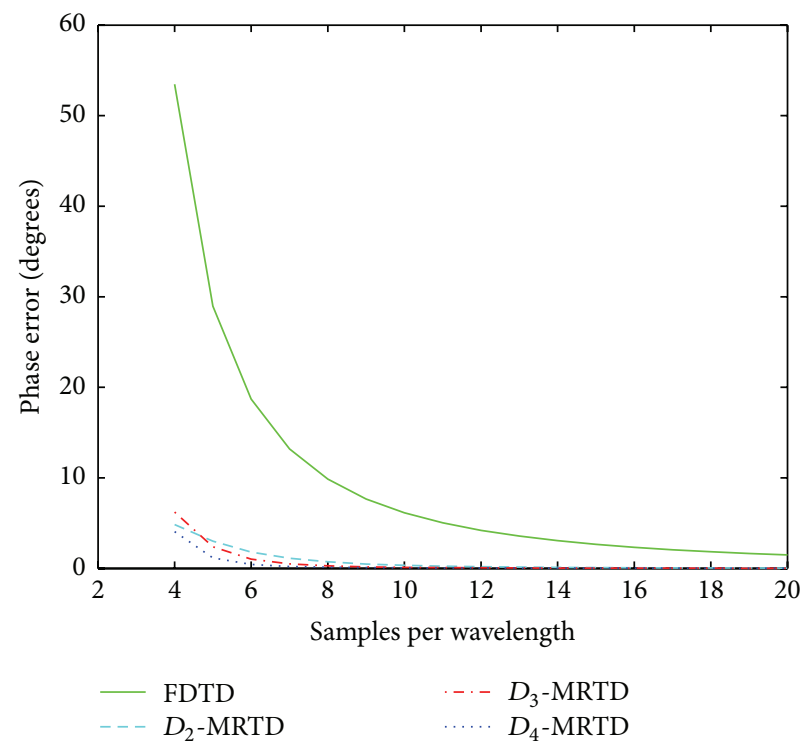

(b)

Figure 5: Phase error (in degrees) for different MRTD schemes. The Courant numbers are $q=0.5$ for (a) and $q=0.1$ for (b).

As we consider the lossless two-conductor transmission lines in (1a) and (1b), the transient values of voltages and currents distributed in space can be Fourier transformed with respect to $k$-coordinates to provide a spectrum of sinusoidal modes. Assuming an eigenmode of the spectral domain with $k_{z}$, the voltages and currents can be written as

$$
\begin{aligned}
V_{K} & =V_{z_{0}} \exp \left[-j\left(k_{z} K \Delta z\right)\right] \\
I_{K+1 / 2} & =I_{z_{0}} \exp \left[-j\left(k_{z}\left(K+\frac{1}{2}\right) \Delta z\right)\right],
\end{aligned}
$$

where $V_{K}$ represents the voltage at point $K \Delta z, I_{K+1 / 2}$ represents the current at point $(K+1 / 2) \Delta z$, and $V_{z_{0}}$ and $I_{z_{0}}$ are the amplitudes of the voltages and currents.

Substituting (40a) and (40b) into (38a) and (38b) and (37a) and (37b), we obtain

$$
\lambda^{2}=-\frac{4}{l c \Delta z^{2}}\left[\sum_{i=0}^{L_{S}-1} a(i) \sin \left(k_{z}\left(i+\frac{1}{2}\right) \Delta z\right)\right]^{2} .
$$

In (41), $\lambda$ is pure imaginary and

$$
|\operatorname{Im}(\lambda)| \leq \frac{2 v}{\Delta z} \sum_{i=0}^{L_{s}-1}|a(i)|,
$$

where $v=1 / \sqrt{l c}$ is the velocity of the wave along with the lines.

Numerical stability is maintained for every spatial mode only when the range of eigenvalues given by (42) is contained entirely within the stable range of time-difference eigenvalues given by (39). Since both ranges are symmetrical around zero, it is adequate to set the upper bound of (42) to be smaller or equal to (39); we can obtain the stability condition

$$
\frac{v \Delta t}{\Delta z} \leq \frac{1}{\sum_{i=0}^{L_{s}-1}|a(i)|} .
$$

Noting the Courant number

$$
q=\frac{v \Delta t}{\Delta z}
$$

the maximum values of $q$ required by a stable algorithm, which are listed in Table 1 as $q_{\max }$, can be calculated from the connection coefficients.

2.5. Dispersion Analysis. To calculate the numerical dispersion, substituting a time harmonic trial solution into (37a) and (37b), we can obtain

$$
\frac{1}{v \Delta t} \sin \left(\frac{\omega \Delta t}{2}\right)=\frac{1}{\Delta z} \sum_{i=0}^{L_{S}^{-1}} a(i) \sin \left(k_{z}\left(i+\frac{1}{2}\right) \Delta z\right),
$$

where $\omega$ is the wave angular frequency and $k_{z}$ is the numerical wave number.

Using the number of cells per wavelength $n_{l}=\lambda_{\mathrm{REAL}} / \Delta z$ and the wave number $k_{z}=(2 \pi) / \lambda_{\mathrm{NUM}}$, we obtain the dispersion relationship

$$
\frac{1}{q} \sin \frac{\pi q}{n_{l}}=\sum_{i=0}^{L_{s}-1} a(i) \sin \left[(2 i+1) \frac{\pi u}{n_{l}}\right],
$$

where $u=\lambda_{\mathrm{REAL}} / \lambda_{\mathrm{NUM}}$ is the ratio between the theoretical and numerical wavelength.

The value of $u$ could be computed by Newton iterative method and using the formula $|(u-1) \times 360|$ calculates the phase error in degrees. Figure 5 shows the phase errors of different MRTD schemes versus the samples per wavelength. Compared with the conventional FDTD method at the same sampling numbers, the MRTD schemes show better numerical dispersion properties than FDTD method, which means the discretization error of the MRTD schemes is smaller than the FDTD method. 


\section{MRTD Scheme for Two-Conductor Lossy Transmission Lines}

In this section, we will extend the MRTD scheme to the twoconductor lossy transmission lines.

3.1. MRTD Formulation. For the lossy case, the twoconductor transmission line equations become

$$
\begin{gathered}
\frac{\partial V(z, t)}{\partial z}+r I(z, t)+l \frac{\partial I(z, t)}{\partial t}=0 \\
\frac{\partial I(z, t)}{\partial z}+g V(z, t)+c \frac{\partial V(z, t)}{\partial t}=0,
\end{gathered}
$$

where $r, l, g$, and $c$ are the per-unit-length resistance, inductance, conductance, and capacitance, respectively.

Compared to the lossless transmission lines, the lossy case must consider the resistance losses along the lines and the losses in the medium. However, the steps to obtain the MRTD scheme are the same. Similar to the lossless case, we firstly extend the voltage and current with Daubechies' scaling functions and the rectangular function and use $V_{k}^{n}$ and $I_{k+1 / 2}^{n+1 / 2}$ representing the voltage at the point $(k \Delta z, n \Delta t)$ and the current at the point $((k+1 / 2) \Delta z,(n+1 / 2) \Delta t)$, respectively, and $\Delta z$ and $\Delta t$ represent the spatial and temporal discretization intervals. By applying the Galerkin technique, we can obtain the iterative equations for the lossy transmission lines equations

$$
\begin{aligned}
V_{k}^{n+1}= & P_{1} V_{k}^{n} \\
& -P_{2} \frac{\Delta t}{\Delta z} c^{-1} \sum_{i=0}^{L_{S}-1} a(i)\left(I_{k+i+1 / 2}^{n+1 / 2}-I_{k-i-1 / 2}^{n+1 / 2}\right) \\
I_{k+1 / 2}^{n+1 / 2}= & Q_{1} I_{k+1 / 2}^{n-1 / 2} \\
& -Q_{2} \frac{\Delta t}{\Delta z} l^{-1} \sum_{i=0}^{L_{S}-1} a(i)\left(V_{k+i+1}^{n}-V_{k-i}^{n}\right)
\end{aligned}
$$

where $a(i)$ is the connection coefficient and $P_{1}, P_{2}, Q_{1}$, and $Q_{2}$ are constants

$$
\begin{aligned}
P_{1} & =\left(1+\frac{\Delta t}{2} g c^{-1}\right)^{-1}\left(1-\frac{\Delta t}{2} g c^{-1}\right) \\
P_{2} & =\left(1+\frac{\Delta t}{2} g c^{-1}\right)^{-1} \\
Q_{1} & =\left(1+\frac{\Delta t}{2} r l^{-1}\right)^{-1}\left(1-\frac{\Delta t}{2} r l^{-1}\right) \\
Q_{2} & =\left(1+\frac{\Delta t}{2} r l^{-1}\right)^{-1} .
\end{aligned}
$$

The differences between (48a) and (48b) and (13a) and (13b) are the coefficients of terms in the iterative equations that are caused by the unit-per-length resistance $r$ and inductance $g$. If we set $r=0$ and $g=0$, the coefficients $P_{1}$, $P_{2}$ and $Q_{1}, Q_{2}$ are all equal to 1 , and the iterative equations (48a) and (48b) will degenerate to iterative equations (13a) and (13b).

For the lossy case, we should also modify the iterative equations at the terminal and near the terminal of the lines. Considering the two-conductor lines shown in Figure 2, we assume the length of the total line is $L$ and the resistive loads are $R_{S}$ and $R_{L}$. The line is divided uniformly into NDZ segments with the space interval $\Delta z$ and the total solution time is divided into NDT steps with the uniform time interval $\Delta t$. Following the same steps in Section 2.2, we could obtain the modified iterative equations.

For the voltage iterative equation at the source

$$
\begin{aligned}
V_{0}^{n+1} & =\left(P_{2} \sum_{i=0}^{L_{S}-1} a(i)+\frac{\Delta z}{\Delta t} c R_{S}\right)^{-1} \\
\cdot & {\left[\left(P_{1} \frac{\Delta z}{\Delta t} c R_{S}-P_{2} \sum_{i=0}^{L_{S}-1} a(i)\right) V_{0}^{n}\right.} \\
- & \left.2 P_{2} R_{S} \sum_{i=0}^{L_{S}-1} a(i) I_{i+1 / 2}^{n+1 / 2}+P_{2} \sum_{i=0}^{L_{S}^{-1}} a(i)\left(V_{S}^{n+1}+V_{S}^{n}\right)\right] .
\end{aligned}
$$

For the voltage iterative equation at the load

$$
\begin{aligned}
& V_{\mathrm{NDZ}}^{n+1}=\left(P_{2} \sum_{i=0}^{L_{S}-1} a(i)+\frac{\Delta z}{\Delta t} c R_{L}\right)^{-1} \\
& \cdot\left[\left(P_{1} \frac{\Delta z}{\Delta t} c R_{L}-P_{2} \sum_{i=0}^{L_{S}-1} a(i)\right) V_{\mathrm{NDZ}}^{n}\right. \\
& +2 P_{2} R_{L} \sum_{i=0}^{L_{S}-1} a(i) I_{\mathrm{NDZ}-i+1 / 2}^{n+1 / 2} \\
& \left.+P_{2} \sum_{i=0}^{L_{S}-1} a(i)\left(V_{L}^{n+1}+V_{L}^{n}\right)\right] .
\end{aligned}
$$

For the voltage iterative equations near the source

$$
\begin{gathered}
V_{k}^{n+1}=P_{1} V_{k}^{n}-P_{2}\left(\sum_{i=0}^{k-1}(2 i+1) a(i)\right)^{-1} \frac{\Delta t}{\Delta z} \\
\cdot c^{-1} \sum_{i=0}^{k-1} a(i)\left(I_{k+i+1 / 2}^{n+1 / 2}-I_{k-i-1 / 2}^{n+1 / 2}\right)
\end{gathered}
$$

for $k=1,2, \ldots, L_{S}-1$

For the voltage iterative equations near the load

$$
\begin{gathered}
V_{k}^{n+1}=P_{1} V_{k}^{n}-P_{2}\left(\sum_{i=0}^{\mathrm{NDZ}-k-1}(2 i+1) a(i)\right)^{-1} \frac{\Delta t}{\Delta z} \\
\cdot c^{-1} \sum_{i=0}^{\mathrm{NDZ}-k-1} a(i)\left(I_{k+i+1 / 2}^{n+1 / 2}-I_{k-i-1 / 2}^{n+1 / 2}\right)
\end{gathered}
$$

for $k=\mathrm{NDZ}-L_{S}+1, \mathrm{NDZ}-L_{S}+2, \ldots, \mathrm{NDZ}-1$. 

(48a)

The voltages at the interior points are determined from

$$
V_{k}^{n+1}=P_{1} V_{k}^{n}-P_{2} \frac{\Delta t}{\Delta z} c^{-1} \sum_{i=0}^{L_{S}-1} a(i)\left(I_{k+i+1 / 2}^{n+1 / 2}-I_{k-i-1 / 2}^{n+1 / 2}\right)
$$

for $k=L_{S}, L_{S}+1, \ldots, \mathrm{NDZ}-L_{S}$.

For the current iterative equations near the source

$$
\begin{gathered}
I_{k+1 / 2}^{n+1 / 2}=Q_{1} I_{k+1 / 2}^{n-1 / 2}-Q_{2}\left(\sum_{i=0}^{k}(2 i+1) a(i)\right)^{-1} \frac{\Delta t}{\Delta z} \\
\cdot l^{-1} \sum_{i=0}^{k} a(i)\left(V_{k+i+1}^{n}-V_{k-i}^{n}\right)
\end{gathered}
$$

for $k=0,1, \ldots, L_{S}-2$.

For the current iterative equations near the load

$$
\begin{gathered}
I_{k+1 / 2}^{n+1 / 2}=Q_{1} I_{k+1 / 2}^{n-1 / 2}-Q_{2}\left(\sum_{i=0}^{\mathrm{NDZ}-k-1}(2 i+1) a(i)\right)^{-1} \frac{\Delta t}{\Delta z} \\
\cdot l^{-1} \sum_{i=0}^{\mathrm{NDZ}-k-1} a(i)\left(V_{k+i+1}^{n}-V_{k-i}^{n}\right)
\end{gathered}
$$

for $k=\mathrm{NDZ}-L_{S}+1, \mathrm{NDZ}-L_{S}+2, \ldots, \mathrm{NDZ}-1$. (48b)

The currents at the interior points are determined from

$$
I_{k+1 / 2}^{n+1 / 2}=Q_{1} I_{k+1 / 2}^{n-1 / 2}-Q_{2} \frac{\Delta t}{\Delta z} l^{-1} \sum_{i=0}^{L_{S}-1} a(i)\left(V_{k+i+1}^{n}-V_{k-i}^{n}\right)
$$

for $k=L_{S}-1, L_{S}, \ldots, \mathrm{NDZ}-L_{S}$.

Besides, for the two-conductor line shown in Figure 4, we can obtain the iterative equation at the load

$$
\begin{aligned}
V_{\mathrm{NDZ}}^{n+1}= & P_{1} V_{\mathrm{NDZ}}^{n} \\
& -P_{2} \frac{\Delta t}{\Delta z} c^{-1} \sum_{i=0}^{L_{S}-1} 2 a(i)\left(I_{\mathrm{NDZ}-i+1 / 2}^{n+1 / 2}-I_{L}^{n+1 / 2}\right),
\end{aligned}
$$

where

$$
\begin{gathered}
I_{L}^{n+1 / 2}=I_{L}^{n-3 / 2}+\frac{\Delta t}{L_{L}}\left(\left(V_{\mathrm{NDZ}}^{n}+V_{\mathrm{NDZ}}^{n-1}\right)\right. \\
\left.-\left(V_{L}^{n}+V_{L}^{n-1}\right)-2 R_{L} I_{L}^{n-1 / 2}\right)
\end{gathered}
$$

for $n=2,3, \ldots$, NDT.
3.2. Stability Analysis. To study the stability of the MRTD scheme for lossy case, we need to make some changes. Rewrite (48a) and (48b) as follows:

$$
\begin{aligned}
& \frac{P_{2}^{-1} V_{k}^{n+1}-P_{2}^{-1} P_{1} V_{k}^{n}}{\Delta t} \\
& =-\frac{1}{c \Delta z} \sum_{i=0}^{L_{S}-1} a(i)\left(I_{k+i+1 / 2}^{n+1 / 2}-I_{k-i-1 / 2}^{n+1 / 2}\right) \\
& \frac{Q_{2}^{-1} I_{k+1 / 2}^{n+1 / 2}-Q_{2}^{-1} Q_{1} I_{k+1 / 2}^{n-1 / 2}}{\Delta t} \\
& =-\frac{1}{l \Delta z} \sum_{i=0}^{L_{S}-1} a(i)\left(V_{k+i+1}^{n}-V_{k-i}^{n}\right) .
\end{aligned}
$$

The finite-difference approximations of the time derivations on the left side of (61a) and (61b) are different from the left side of (37a) and (37b), but we can also write them as an eigenvalue problem

$$
\begin{aligned}
\frac{P_{2}^{-1} V_{k}^{n+1}-P_{2}^{-1} P_{1} V_{k}^{n}}{\Delta t} & =\lambda V_{k}^{n+1 / 2} \\
\frac{Q_{2}^{-1} I_{k+1 / 2}^{n+1 / 2}-Q_{2}^{-1} Q_{1} I_{k+1 / 2}^{n-1 / 2}}{\Delta t} & =\lambda I_{k+1 / 2}^{n} .
\end{aligned}
$$

In order to avoid instability during normal time stepping, the imaginary part of $\lambda$ also must satisfy

$$
|\operatorname{Im}(\lambda)| \leq \frac{2}{\Delta t}
$$

Then following the steps in Section 2.4, using the Fourier transform, expand the transient values of voltages and currents distributed in space. We can also obtain the same stability condition as (43)

$$
\frac{v \Delta t}{\Delta z} \leq \frac{1}{\sum_{i=0}^{L_{s}-1}|a(i)|}
$$

However, the dispersion analysis for the iterative equations of the lossy transmission lines is quite different from the lossless case. Since $P_{2}^{-1} \neq P_{2}^{-1} P_{1}$ and $Q_{2}^{-1} \neq Q_{2}^{-1} Q_{1}$, that is, the coefficient of $V_{k}^{n+1}$ is not equal to the coefficient of $V_{k}^{n}$ and the coefficient of $I_{k+1 / 2}^{n+1 / 2}$ is not equal to the coefficient of $I_{k+1 / 2}^{n-1 / 2}$ on the left side of (61a) and (61b), if we substitute a time harmonic into the iterative equations into (61a) and (61b), there are some other terms that could affect the ratio between the theoretical and numerical wavelength except samples per wavelength, so we could not obtain a brief dispersion relation as (45). However, the numerical results in Section 4.2 show that the MRTD method could obtain a more accurate result than the FDTD method at the same space interval and time interval. 


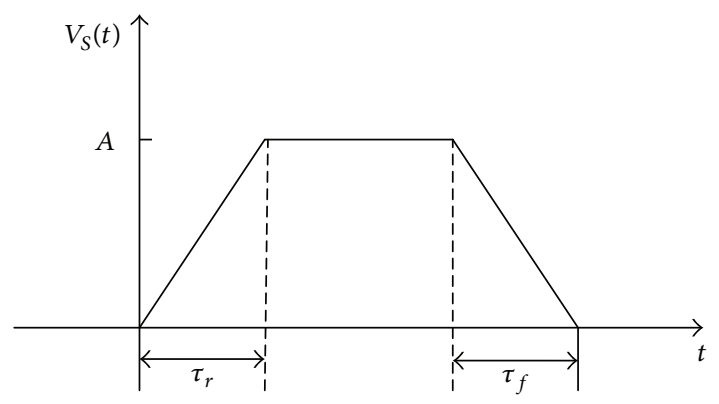

FIgURE 6: Trapezoidal pulse with the rise time $\tau_{r}$ and fall time $\tau_{f}$.

\section{Numerical Result}

4.1. Lossless Transmission Lines. In this section, the twoconductor lossless transmission lines as shown in Figure 2 are considered to calculate the terminal voltages. The length of the lines is $L=400 \mathrm{~m}$ and the per-unit-length capacitance and inductance are $c=100 \mathrm{pF} / \mathrm{m}$ and $l=0.25 \mu \mathrm{H} / \mathrm{m}$, respectively. This corresponds to RG58U coaxial cable [18]. The characteristic impedance of the line is $Z_{C}=\sqrt{l / c}=50 \Omega$ and the velocity of propagation is $v=1 / \sqrt{l c}=200 \mathrm{~m} / \mu \mathrm{s}$. The terminal load is $100 \Omega$ and the source resistance is $50 \Omega$.

We will use a trapezoidal pulse as shown in Figure 6 as the source voltage, whose initial value is $0 \mathrm{~V}$ and amplitude is $A=30 \mathrm{~V}$, total time is $10 \mu \mathrm{s}$, rise time is $\tau_{r}=1 \mu \mathrm{s}$, and fall time is $\tau_{f}=1 \mu \mathrm{s}$, respectively. The bandwidth of the pulse is approximate to $\mathrm{BW}=1 / \tau_{r}$, so the segment length $\Delta z$ should be electrically short at this frequency requiring $\Delta z \leq(1 / 10)\left(v / f_{\max }\right)\left(f_{\max }=1 / \tau_{r}\right)[18]$. As we take the rise time of the trapezoidal pulse to be $1 \mu \mathrm{s}$, the approximate bandwidth of the pulse is $1 \mathrm{MHz}$ and the maximum section length of $\Delta z$ should be less than $20 \mathrm{~m}$, noting $\Delta z_{\max }=20$. Since we divide the line into NDZ segments uniformly, NDZ should be greater than $L / \Delta z_{\max }=20$. The time step will be calculated by (44) with different Courant number $q$.

Under the conditions of the space discretization number $\mathrm{NDZ}=20$ and the Courant number $q=0.5$, we calculate the terminal voltages using the MRTD schemes with $D_{2}$, $D_{3}$, and $D_{4}$ wavelets' scaling functions. We also calculate the terminal voltages by the FDTD method under the same conditions. Figure 7 shows the numerical results by different methods and $D_{i}$-MRTD represents the MRTD scheme using Daubechies' scaling functions with $i$ vanishing moment as basis functions, where $i=2,3,4$.

For the time-dependent discrete terminal voltages, the relative error is defined as follows [23]:

$$
\epsilon=\frac{\sum_{i=1}^{\mathrm{NDT}}(x(i)-\widehat{x}(i))^{2}}{\sum_{i=1}^{\mathrm{NDT}} \hat{x}^{2}(i)} .
$$

Here, $x(i)$ represents the numerical results of FDTD and MRTD at each time discretization point and $\widehat{x}(i)$ is the numerical result of series solution at each time discretization point, which can be regarded as the exact solution [18]. Taking $\widehat{x}(i)$ as the exact result, we calculate the relative errors of FDTD method and MRTD scheme.

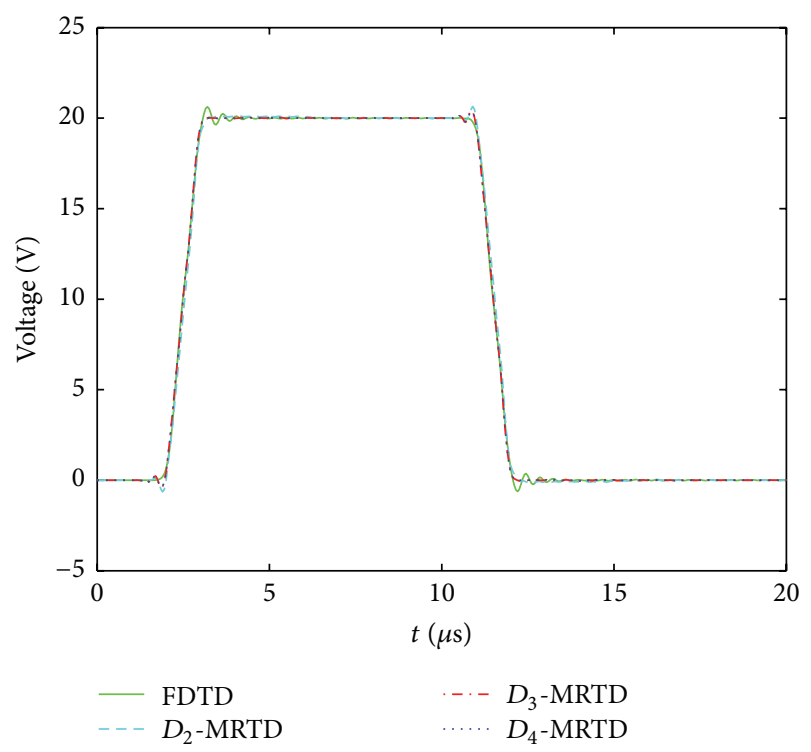

FIGURE 7: Terminal voltage calculated by different numerical methods. The space discretization number and the Courant number are $\mathrm{NDZ}=20$ and $q=0.5$.

TABLE 2: Relative errors and runtime of different methods (NDZ = $20, q=0.5)$.

\begin{tabular}{lcc}
\hline Analysis scheme & Relative error & Runtime (s) \\
\hline FDTD & $1.08 \%$ & 0.0011 \\
$D_{2}$-MRTD & $1.93 \%$ & 0.0050 \\
$D_{3}$-MRTD & $0.66 \%$ & 0.0054 \\
$D_{4}$-MRTD & $0.62 \%$ & 0.0058 \\
\hline
\end{tabular}

Table 2 shows the relative errors and runtime for different schemes. Since the iterative equations in MRTD schemes contain more terms than the conventional FDTD method, the MRTD schemes expend more runtime. When we use $D_{2}$-MRTD, the numerical result shows a larger relative error. The reason is that the vanishing moment of the $D_{2}$ wavelet's scaling function is not high enough. For the wavelets' scaling functions whose vanishing moment is high enough, like $D_{3}$ wavelet and $D_{4}$ wavelet, the numerical results show smaller relative errors.

Figure 8 shows the relative errors versus the space discretization numbers. The space interval will decrease with the increase of the space discretization number. We can see from the figures that the relative errors for $D_{2}$-MRTD increase with the increase of NDZ. And, for $D_{3}$-MRTD and $D_{4}$-MRTD, the relative errors decrease with the increase of NDZ and show a little smaller relative error than the conventional FDTD. It means we can get a more accurate result at same space interval and time interval by $D_{3}$-MRTD and $D_{4}$-MRTD.

Figure 9 describes the relative errors versus the Courant numbers. The time interval will decrease with the decrease of the Courant number. The results show that, with the decrease of the Courant numbers, the relative errors are almost unchanged for the conventional FDTD method, while the relative errors are quite different with different Courant 


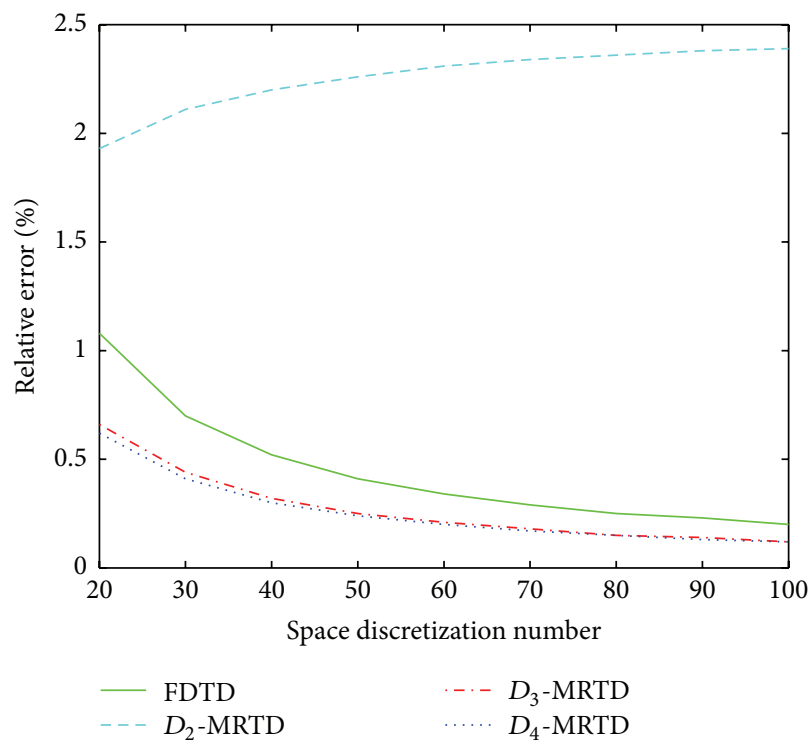

(a)

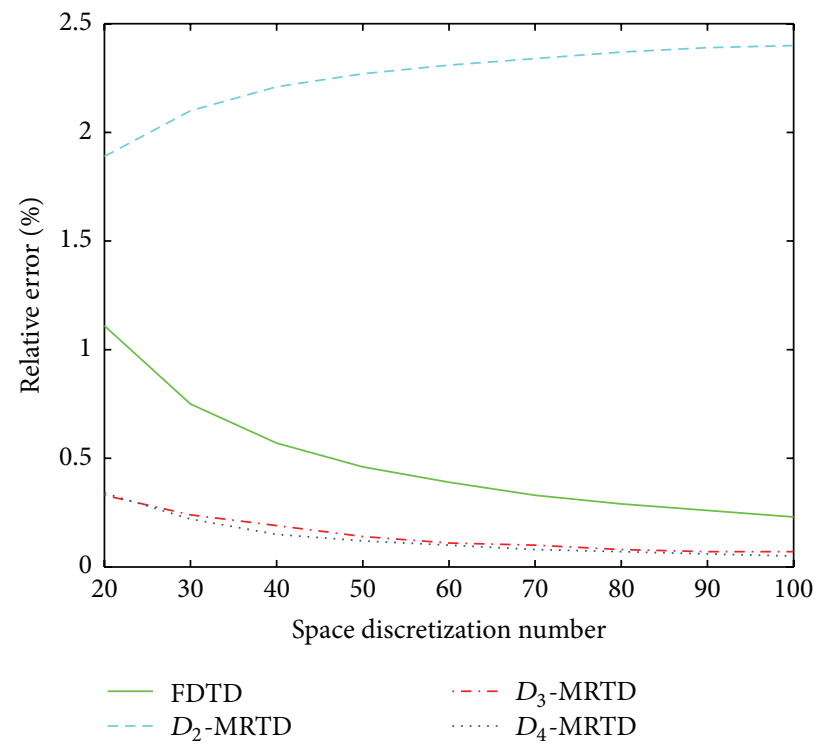

(b)

Figure 8: Relative error for different discretization numbers. The Courant numbers are $q=0.5$ for (a) and $q=0.1$ for (b).

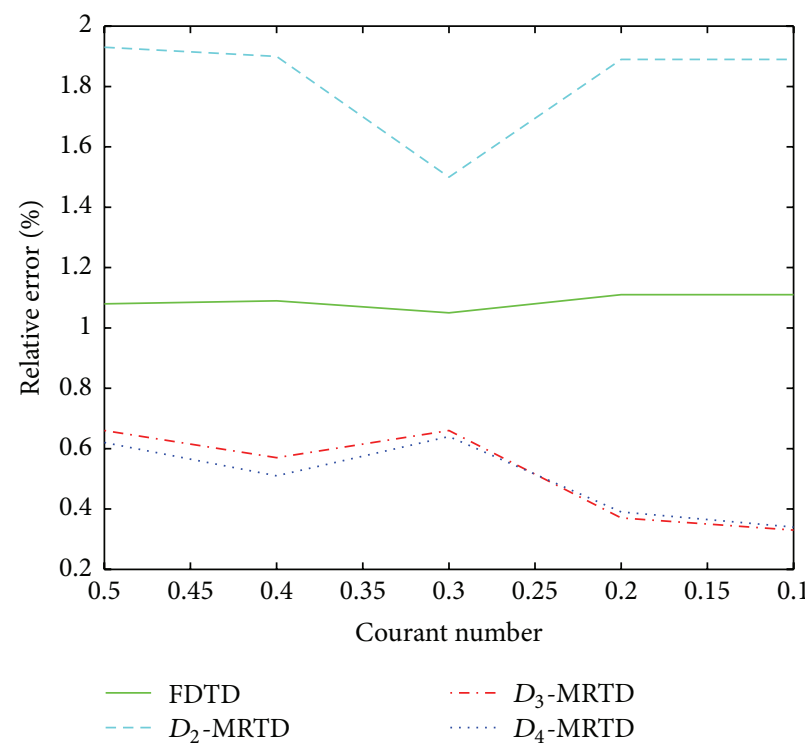

(a)

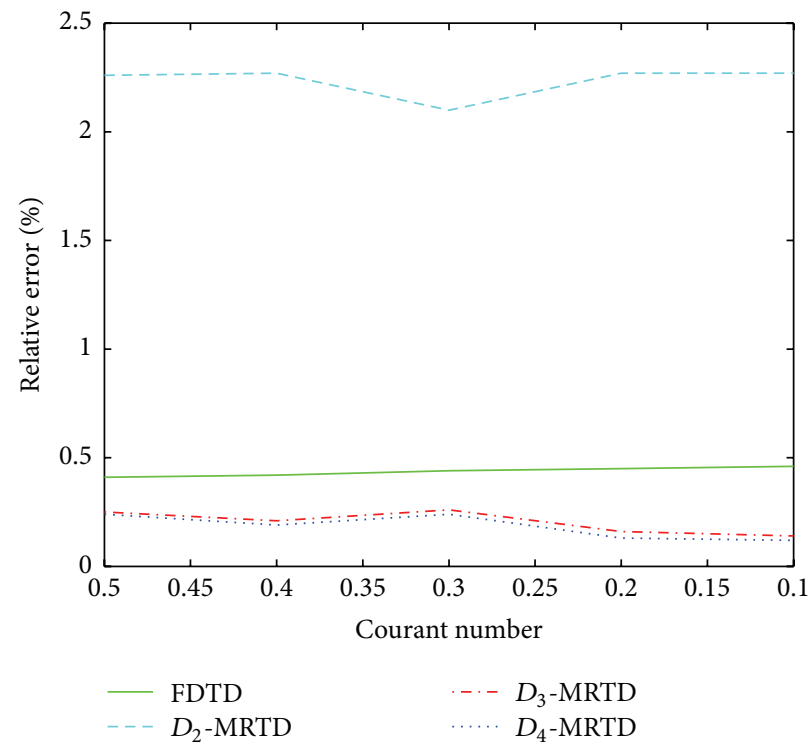

(b)

FIGURE 9: Relative errors for different Courant numbers. The space discretization numbers are NDZ $=20$ for (a) and NDZ = 50 for (b).

numbers for all the three MRTD schemes. That means the choice of the Courant number significantly affects the relative errors of the MRTD schemes, and we could choose the proper Courant number to optimize the MRTD schemes.

To validate the stability of the MRTD schemes, we increase the initial value of the pulse to $20 \mathrm{~V}$ and keep other parameters unchanged. Figure 10 shows the relative errors versus the pace discretization numbers. It can be seen from the figures that the $D_{2}$-MRTD scheme shows a larger relative error and the relative errors of $D_{3}$-MRTD and $D_{4}$ MRTD schemes are smaller than the conventional FDTD method.
The numerical results for the lossless transmission lines also show that the $D_{2}$-MRTD does not perform better than the FDTD method; meanwhile, $D_{3}$-MRTD and $D_{4}$-MRTD schemes show better quality in accuracy and stability. The reason for this phenomenon is that the scaling function of the $\mathrm{D}_{2}$ wavelet does not have enough high vanishing moment. When we use Daubechies' scaling functions to expand the voltages and currents in the two-conductor transmission lines equations, the vanishing moment decides the accuracy of the approximation. The scaling functions with high vanishing moments could approximate voltages and currents more accurately; however, the scaling functions with low 


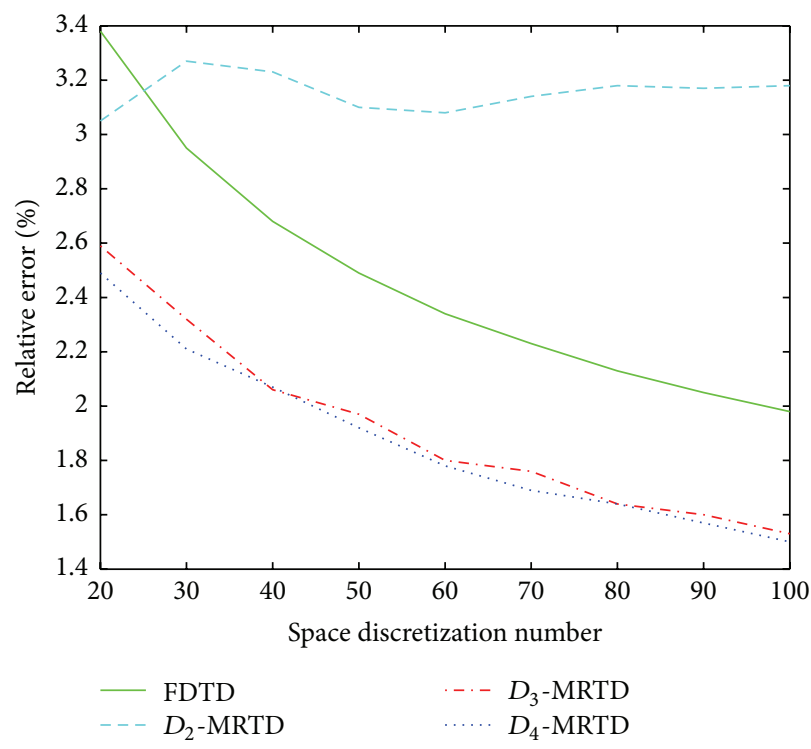

(a)

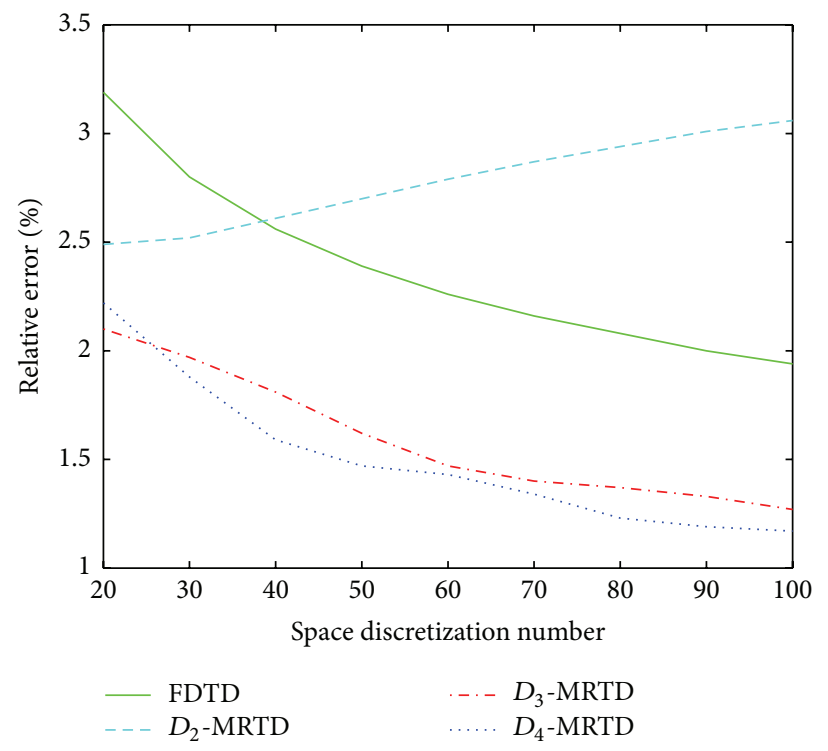

(b)

Figure 10: Relative error for different space discretization numbers for the initial value of the trapezoidal pulse to be $20 \mathrm{~V}$. The Courant numbers are $q=0.5$ for (a) and $q=0.1$ for (b).

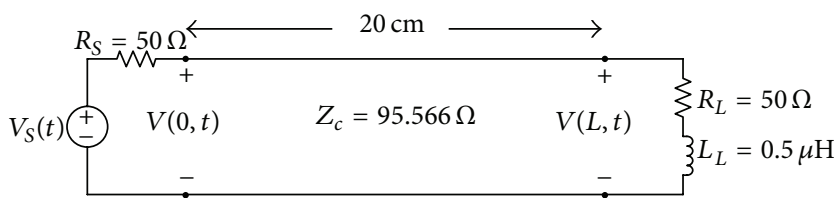

(a)

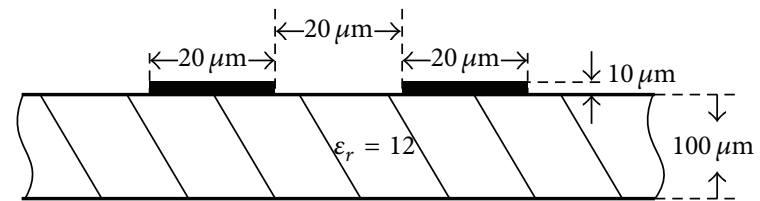

(b)

FIGURE 11: A lossy printed circuit board: (a) line dimensions and terminations and (b) cross-sectional dimensions.

vanishing moments like $D_{2}$ wavelet's may introduce a larger error. So we can see from Figure 5 that $D_{2}$-MRTD has a better numerical dispersion property than the FDTD method, but it gets larger relative errors in numerical computation. For the scaling functions which have high enough vanishing moments like $D_{3}$ wavelet and $D_{4}$ wavelet, the numerical results show smaller relative errors and are in agreement with the dispersion analysis. However, a high vanishing moment for scaling function may increase the computation complexity in MRTD schemes. So the vanishing moments of the wavelet's scaling functions have a great effect on the accuracy of the MRTD scheme; it is necessary to choose a scaling function with proper vanishing moment when we use the MRTD scheme for the numerical computation.

4.2. Lossy Transmission Lines. In this section, we will consider the lossy two-conductor transmission lines shown in Figure 11. Two conductors of rectangular cross section of width $w=20 \mu \mathrm{m}$ and thickness $t=10 \mu \mathrm{m}$ are separated by $s=20 \mu \mathrm{m}$ and placed on one side of a silicon substrate $\left(\varepsilon_{r}=12\right)$ of thickness $h=100 \mu \mathrm{m}$; the total line length is $L=20 \mathrm{~cm}$. The near end is a source with a $R_{S}=$ $50 \Omega$ resistance and the far end is a load with a $R_{L}=50 \Omega$ resistance and $L_{L}=0.5 \mu \mathrm{H}$ inductance in series. The perunit-length inductance and capacitance were computed as $l=$ $0.805969 \mu \mathrm{H} / \mathrm{m}$ and $c=88.2488 \mathrm{pF} / \mathrm{m}$. This gives a velocity of

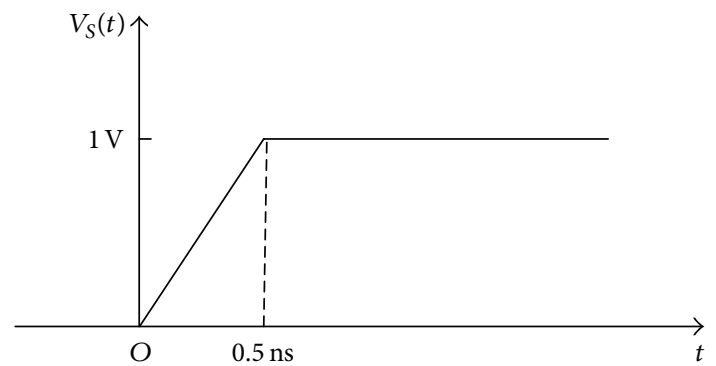

FIGURE 12: Representation of the source voltage waveform.

$v=1.18573 \times 10^{8} \mathrm{~m} / \mathrm{s}$ and a one-way time delay of $T_{D}=1.68672 \mathrm{~ns}$, which gives an effective dielectric constant of $\left(\varepsilon_{r}^{\prime}=6.4\right)$ and characteristic impedance of $Z_{C}=$ $95.566 \Omega$. The per-unit-length dc resistance is computed as $r=1 /(\sigma w t)=86.207 \Omega / \mathrm{m}$ [18]. Dielectric loss is not included in these calculations, which means the per-unitlength conductance is $g=0$.

The source is a ramp function as shown in Figure 12, the initial value of the ramp function is $0 \mathrm{~V}$, and the amplitude is $A=1 \mathrm{~V}$ with a rise time of $\tau_{r}=0.5 \mathrm{~ns}$. The total computing time is $20 \mathrm{~ns}$. The bandwidth of the source is approximate to $\mathrm{BW}=1 / \tau_{r}=2 \mathrm{GHz}$. The space discretization step for the MRTD was chosen to be $\lambda / 10$, so the space discretization 


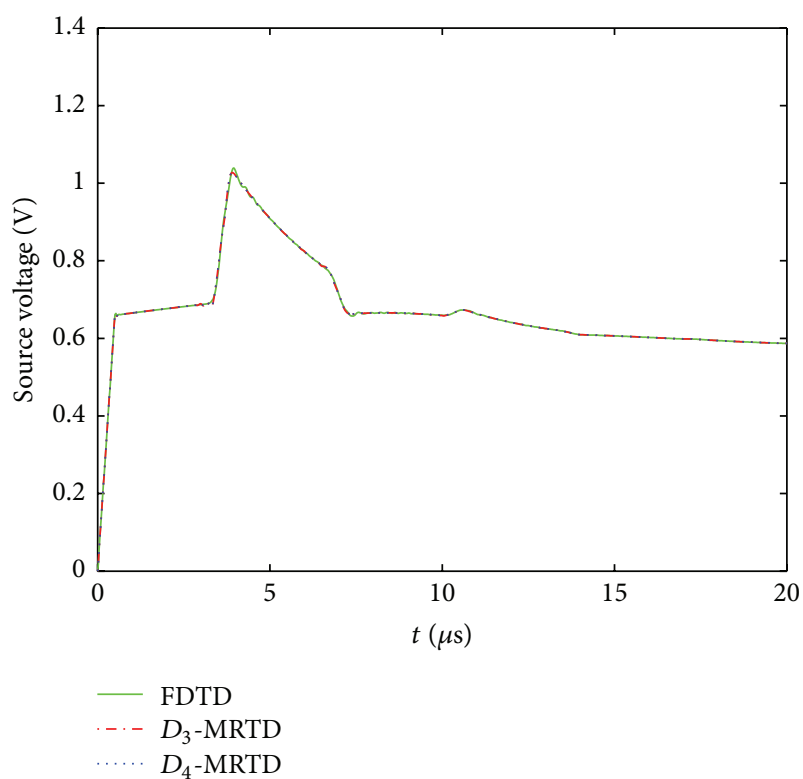

(a)

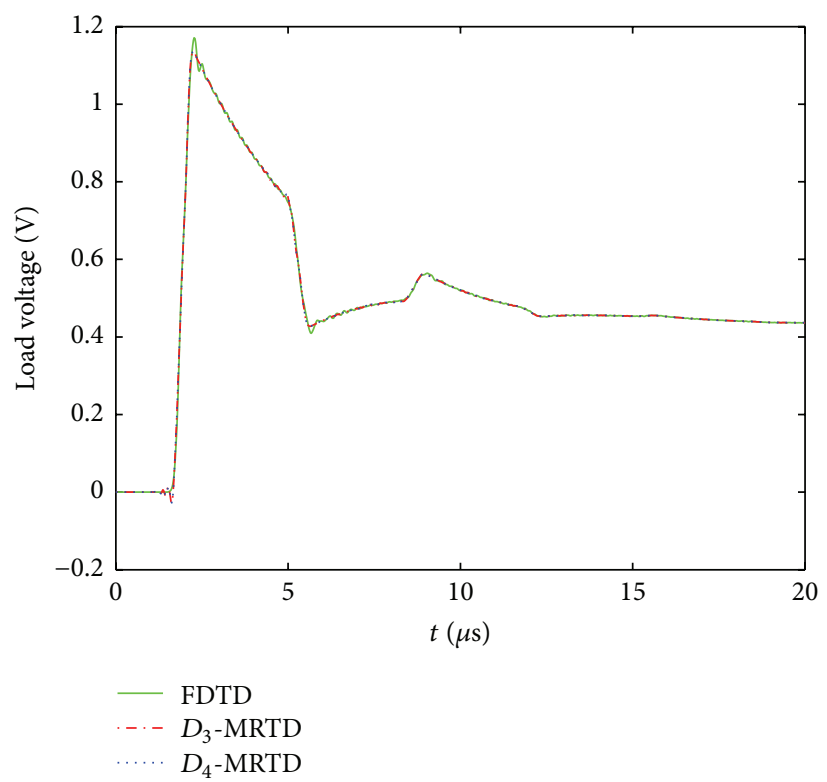

(b)

FIgURE 13: Terminal voltages for lossy transmission lines: (a) for the near end and (b) for the far end. The space discretization number is $\mathrm{NDZ}=40$ and the Courant number is $q=0.5$.

TABLE 3: Relative errors and runtime of different methods. $V_{0}$ represents the near end voltage and $V_{L}$ represents the far end voltage $(\mathrm{NDZ}=40, q=0.5)$.

\begin{tabular}{lccc}
\hline $\begin{array}{l}\text { Analysis } \\
\text { scheme }\end{array}$ & $\begin{array}{c}\text { Relative error } \\
\text { for } V_{0}\end{array}$ & $\begin{array}{c}\text { Relative error } \\
\text { for } V_{L}\end{array}$ & Runtime (s) \\
\hline FDTD & $0.30 \%$ & $0.92 \%$ & 0.0066 \\
$D_{3}$-MRTD & $0.18 \%$ & $0.57 \%$ & 0.0245 \\
$D_{4}$-MRTD & $0.17 \%$ & $0.54 \%$ & 0.0275 \\
\hline
\end{tabular}

number NDZ should be greater than 34 . The time step will also be calculated by (44) with different Courant numbers.

We calculate the near end voltage and the far end voltage of the lossy $\mathrm{PCB}$ with space discretization number NDZ = 40 and the Courant number $q=0.5$. Figure 13 shows the computing results. Since $D_{2}$-MRTD may introduce a larger error in the computation as shown in Section 4.1, we use $D_{3}$ MRTD and $D_{4}$-MRTD to compute the terminal voltages.

Here, we choose the time-domain to frequency-domain transformation method (TDFD), which is a straightforward adaptation of a common analysis technique for lumped, linear circuits and systems [18], to validate the computing results of MRTD schemes and FDTD method. Table 3 shows the relative errors and the runtime of the MRTD schemes and FDTD method. It can be seen that MRTD schemes spend much time to obtain more accurate results.

Figure 14 describes the relative errors versus the space discretization numbers. For both $D_{3}$-MRTD and $D_{4}$-MRTD, the MRTD schemes show a little smaller relative error than the conventional FDTD. That means the MRTD schemes could obtain a more accurate solution under the same time interval and space interval. This is because $D_{3}$-MRTD and $D_{4}$-MRTD have better dispersion property than the FDTD method and the scaling functions of $D_{3}$ wavelet and $D_{4}$ wavelet have high enough vanishing moments.

Figure 15 shows the relative errors versus the Courant numbers. With the decrease of the Courant number, the relative errors of the FDTD method increase, because the time-domain is oversampled for the FDTD method. However, the MRTD schemes perform decreasing relative errors. That means the MRTD schemes could obtain a more accurate result with a smaller time interval. And even when the FDTD method is oversampled in the time-domain, the MRTD schemes perform well.

\section{Conclusion}

In this paper, we derived the MRTD scheme for the twoconductor transmission lines and studied the stability and the numerical dispersion of this scheme. By viewing the MRTD schemes as the weighted mean of the conventional FDTD method at different space interval, we derived the iterative equations for the terminal voltages when the terminals are pure resistive, and a method is proposed to update the iterative equations which contain some terms whose indices exceed the index range in the MRTD scheme. Using the same method, we derived the iterative equation for the inductive load. Then we extended the MRTD scheme to the lossy transmission lines. Using different wavelets' scaling functions as basis functions, the MRTD schemes are implemented for both lossless case and lossy case and the numerical results are compared to the conventional FDTD method. The numerical results show the MRTD schemes need more runtime to obtain more accurate results. And the vanishing moment of the wavelet's scaling functions will significantly affect the quality of the MRTD scheme; using a scaling function with a proper vanishing moment as basis function in MRTD scheme could obtain a more accurate result. 


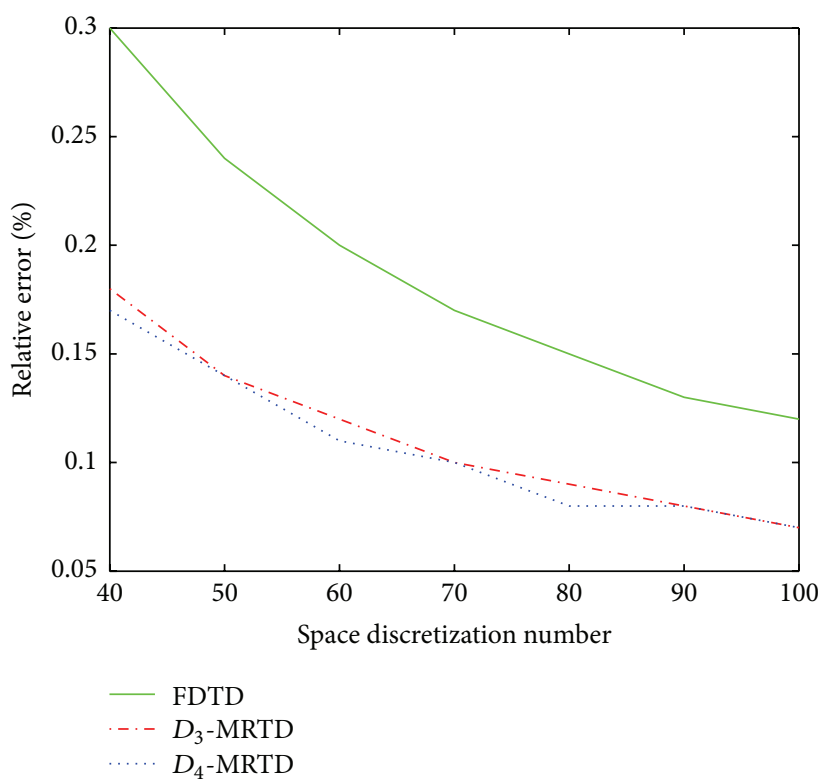

(a)

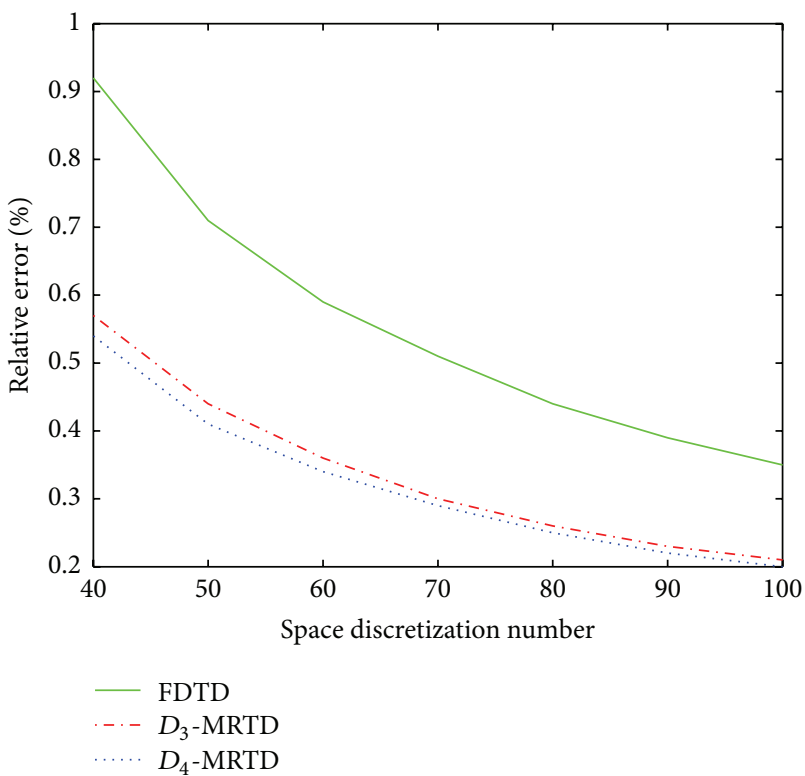

(b)

FIGURE 14: Relative error for the terminal voltages with different space discretization numbers: (a) for the near end and (b) for the far end. The Courant number is $q=0.5$.

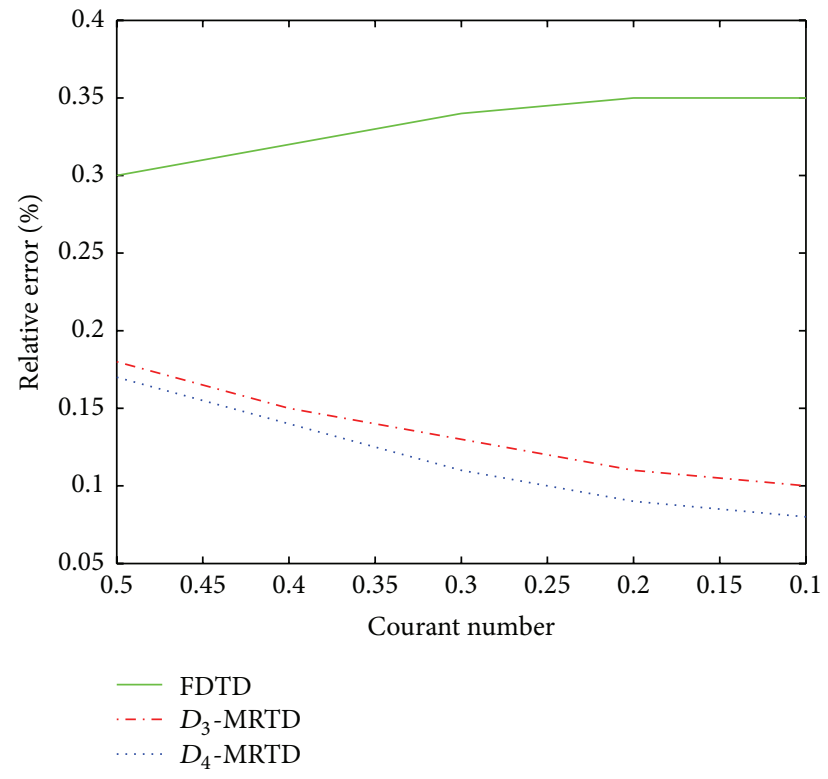

(a)

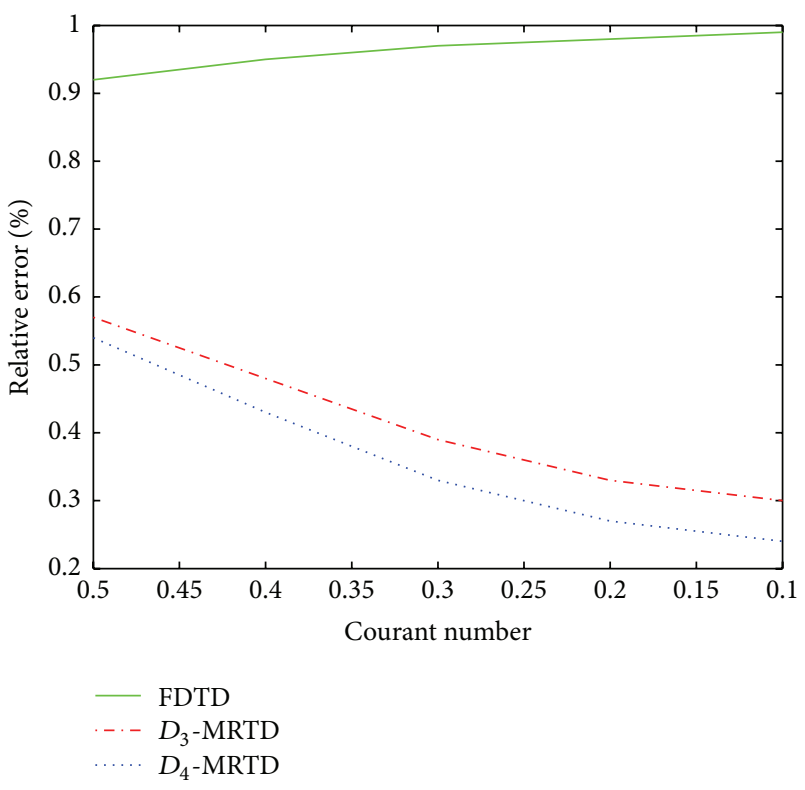

(b)

FIgURE 15: Relative errors for the terminal voltages with different Courant numbers: (a) for the near end and (b) for the far end. The space discretization number is $\mathrm{NDZ}=40$.

\section{Competing Interests}

The authors declare that they have no competing interests.

\section{Acknowledgments}

The authors would like to thank Yinkun Wang for his comments on the paper. This work was supported by the National Natural Science Foundation of China (no. 11271370).

\section{References}

[1] M. Krumpholz and L. P. B. Katehi, "MRTD: new time-domain schemes based on multiresolution analysis," IEEE Transactions on Microwave Theory and Techniques, vol. 44, no. 4, pp. 555-571, 1996.

[2] M. Fujii and W. J. R. Hoefer, "A three-dimensional Harrwavelet-based multi-resolution analysis similar to the 3-D FDTD method-derivation and application," The IEEE Transactions on Microwave Theory and Techniques, vol. 46, no. 12, pp. 2463-2475, 1998. 
[3] Y. W. Cheong, Y. M. Lee, K. H. Ra, J. G. Kang, and C. C. Shin, "Wavelet-Galerkin scheme of time-dependent inhomogeneous electromagnetic problems," IEEE Microwave and Wireless Components Letters, vol. 9, no. 8, pp. 297-299, 1999.

[4] T. Dogaru and L. Carin, "Multiresolution time-domain using CDF biorthogonal wavelets," IEEE Transactions on Microwave Theory and Techniques, vol. 49, no. 5, pp. 902-912, 2001.

[5] X. C. Wei, E. P. Li, and C. H. Liang, "A new MRTD scheme based on Coifman scaling functions for the solution of scattering problems," IEEE Microwave and Wireless Components Letters, vol. 12, no. 10, pp. 392-394, 2002.

[6] E. M. Tentzeris, R. L. Robertson, J. F. Harvey, and L. P. B. Katehi, "Stability and dispersion analysis of battle-lemariebased MRTD schemes," The IEEE Transactions on Microwave Theory and Techniques, vol. 47, no. 7, pp. 1004-1013, 1999.

[7] S. Grivet-Talocia, "On the accuracy of haar-based multiresolution time-domain schemes," IEEE Microwave and Wireless Components Letters, vol. 10, no. 10, pp. 397-399, 2000.

[8] M. Fujii and W. J. R. Hoefer, "Dispersion of time domain wavelet galerkin method based on Daubechies' compactly supported scaling functions with three and four vanishing moments," IEEE Microwave and Wireless Components Letters, vol. 10, no. 4, pp. 125-127, 2000.

[9] A. Alighanbari and C. D. Sarris, "Dispersion properties and applications of the Coifman scaling function based S-MRTD," IEEE Transactions on Antennas and Propagation, vol. 54, no. 8, pp. 2316-2325, 2006.

[10] K. L. Shlager and J. B. Schneider, "Comparison of the dispersion properties of several low-dispersion finite-difference time-domain algorithms," IEEE Transactions on Antennas and Propagation, vol. 51, no. 3, pp. 642-653, 2003.

[11] E. M. Tentzeris, R. L. Robertson, J. F. Harvey, and L. P. B. Katehi, "PML absorbing boundary conditions for the characterization of open microwave circuit components using multiresolution time-domain techniques (MRTD)," IEEE Transactions on Antennas and Propagation, vol. 47, no. 11, pp. 1709-1715, 1999.

[12] Q. S. Cao, Y. C. Chen, and R. Mittra, "Multiple image technique (MIT) and anisotropic perfectly matched layer (APML) in implementation of MRTD scheme for boundary truncations of microwave structures," IEEE Transactions on Microwave Theory and Techniques, vol. 50, no. 6, pp. 1578-1589, 2002.

[13] Y. W. Liu, Y. W. Chen, B. Chen, and X. Xu, "A cylindrical MRTD algorithm with PML and quasi-PML," IEEE Transactions on Microwave Theory and Techniques, vol. 61, no. 3, pp. 1006-1017, 2013.

[14] C. D. Taylor, R. S. Satterwhite, and C. W. Harrison, "The response of a terminated two-wire transmission line excited by a nonuniform electromagnetic field," IEEE Transactions on Antennas and Propagation, vol. 13, no. 6, pp. 987-989, 1965.

[15] A. K. Agrawal, H. J. Price, and S. H. Gurbaxani, "Transient response of multiconductor transmission lines excited by a nonuniform electromagnetic field," IEEE Transactions on Electromagnetic Compatibility, vol. 22, no. 2, pp. 119-129, 1980.

[16] F. Rachidi, "Formulation of the field-to-transmission line coupling equations in terms of magnetic excitation field," IEEE Transactions on Electromagnetic Compatibility, vol. 35, no. 3, pp. 404-407, 1993.

[17] F. Rachidi and S. V. Tkachenko, Electromagnetic Field Interaction with Transmission Lines: From Classical Theory to HF Radiation Effects, vol. 1, WIT Press, 2008.

[18] C. R. Paul, Analysis of Multiconductor Transmission Lines, John Wiley and Sons, New Jersey, NJ, USA, 2nd edition, 2008.
[19] W. Sweldens and R. Piessens, "Wavelet sampling techniques," in Proceedings of the Statistical Computing Section, pp. 20-29, American Statistical Association, 1993.

[20] I. Daubechies, Ten Lectures on Wavelets, SIAM Press, Philadelphia, Pa, USA, 1992.

[21] X. Zhu, T. Dogaru, and L. Carin, "Analysis of the CDF biorthogonal MRTD method with application to PEC targets," IEEE Transactions on Microwave Theory and Techniques, vol. 51, no. 9, pp. 2015-2022, 2003.

[22] A. Taflove, Computational Electrodynamics: The FiniteDifference Time-Domain Method, Artech House, Norwood, NJ, USA, 1995.

[23] A. Alighanbari and C. D. Sarris, "Rigorous and efficient timedomain modeling of electromagnetic wave propagation and fading statistics in indoor wireless channels," IEEE Transactions on Antennas and Propagation, vol. 55, no. 8, pp. 2373-2381, 2007. 


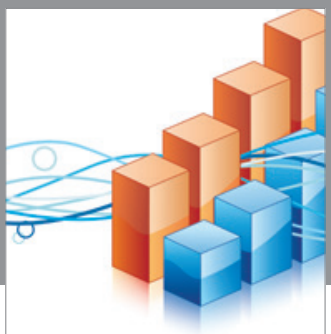

Advances in

Operations Research

vatem alat4

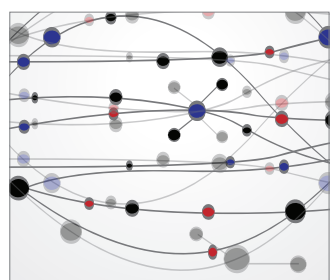

\section{The Scientific} World Journal
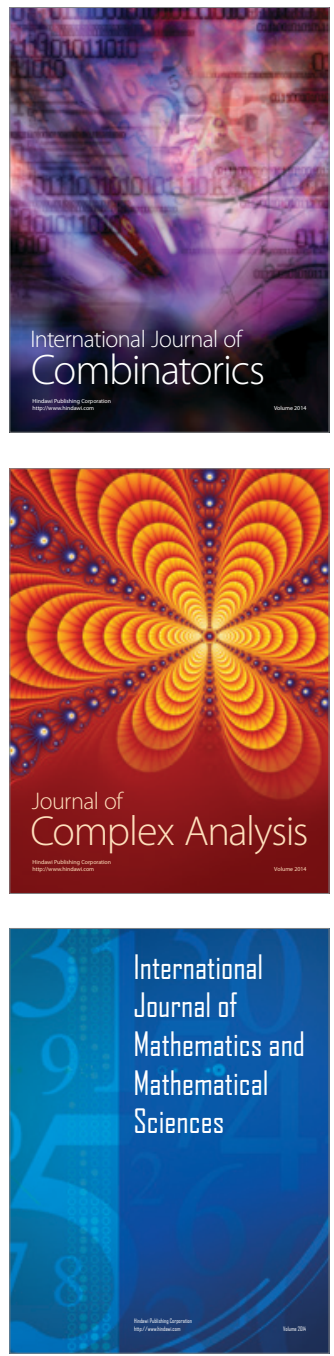
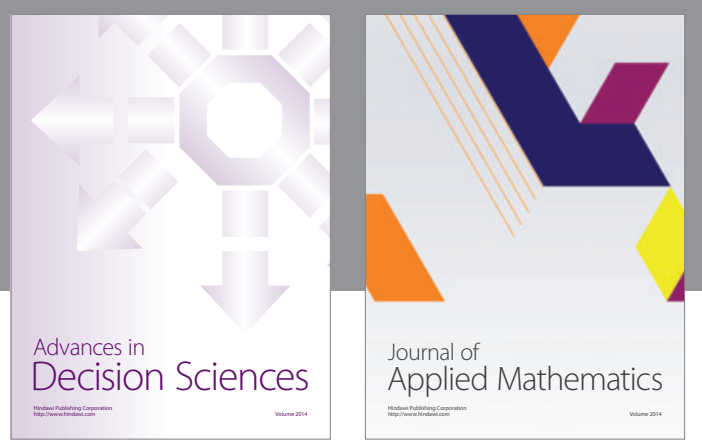

Algebra

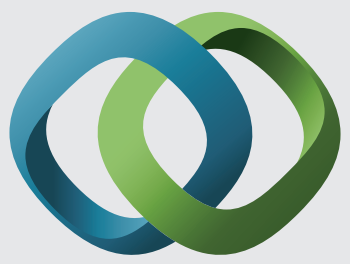

\section{Hindawi}

Submit your manuscripts at

http://www.hindawi.com
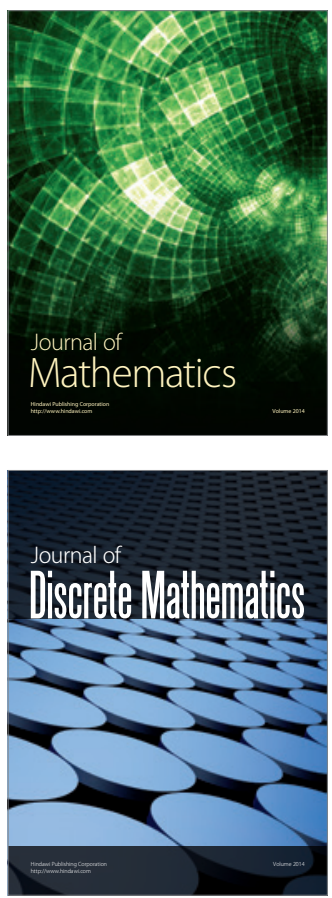

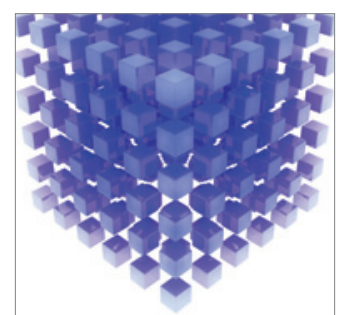

Mathematical Problems in Engineering
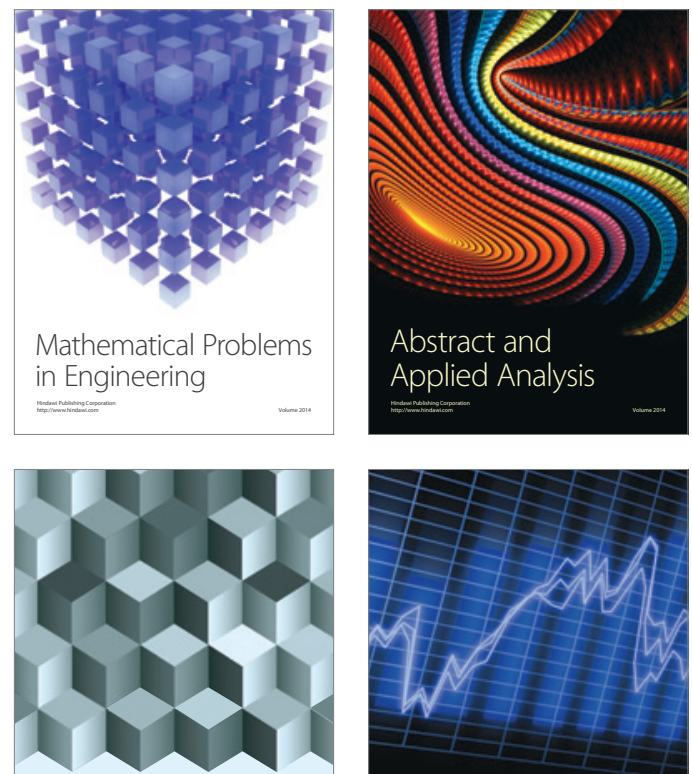

Journal of

Function Spaces

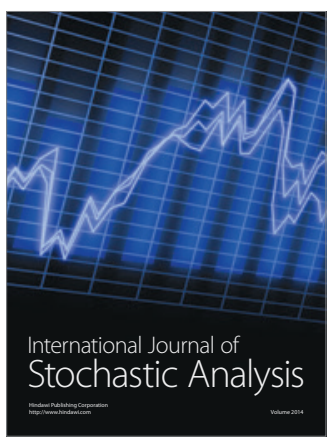

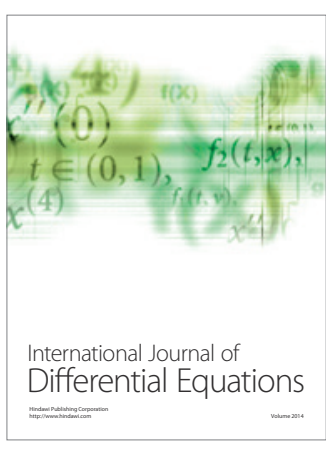
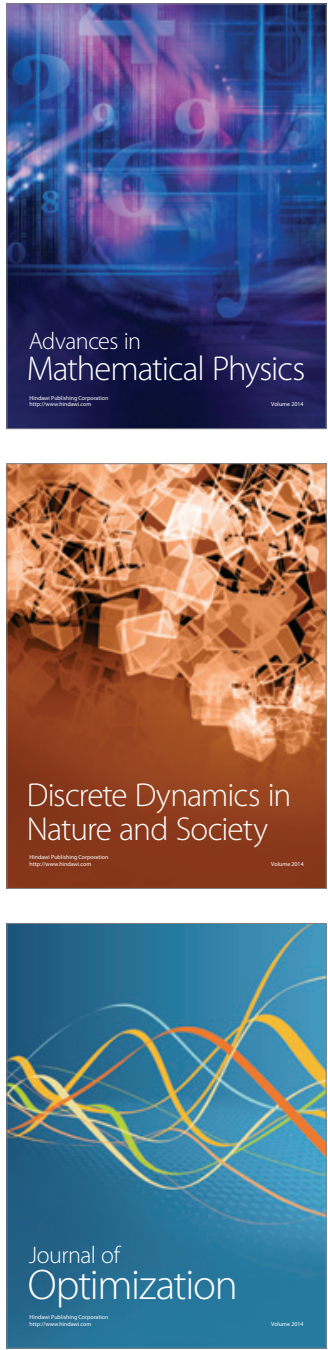